\title{
Three-dimensional prediction of maize pollen dispersal and cross-pollination, and the effects of windbreaks
}

\author{
Tomoki USHIYAMA $^{1 *}$, Mingyuan Du ${ }^{1}$, Satoshi INOUE ${ }^{1}$, Hiroyuki SHIBAIKE ${ }^{1}$, Seiichiro YonemurA ${ }^{1}$, Shigeto KAWASHIMA ${ }^{2}$ \\ and Katsuki AMANO ${ }^{3}$ \\ 1 The National Institute for Agro-Environmental Sciences, Tsukuba, Ibaraki, Japan \\ 2 Graduate School of Agriculture, Kyoto University, Japan \\ 3 The National Center for Seeds and Seedlings, Japan
}

With the extensive adoption of transgenic crops, an understanding of transgene flow is essential to manage gene flow to non-GM crops. Thus, a flexible and accurate numerical model is required to assess gene flow through pollen dispersal. A three-dimensional atmospheric model combined with a diffusion transport model would be a useful tool for predicting pollen dispersal since it would be flexible enough to incorporate the effects of factors such as the spatial arrangement of crop combinations, land use, topography, windbreaks, and buildings. We applied such a model to field measurements of gene flow between two adjacent maize (Zea mays) cultivars, with suppression effects due to windbreaks, in an experimental cornfield in Japan. This combined model reproduced the measured cross-pollination distribution quite well in the case of maize plots with plant windbreaks slightly taller than the maize and without windbreaks, but the model underestimated the effect of a 6-m-tall windbreak net beyond $25 \mathrm{~m}$ from the donor pollen source on cross-pollination. The underestimation was most probably due to the problem of assimilated wind data. The model showed that the 6-m-tall windbreak and the plant wind break suppressed average cross-pollination rate by about $60 \%$ and $30 \%$, respectively. Halftall and coarser mesh windbreak net suppressed cross-pollination rates by $\mathbf{4 0} \%$ by reducing the swirl of donor pollen by reduced wind speed.

Keywords: pollen dispersal / numerical prediction / cross-pollination / maize / windbreak

\section{INTRODUCTION}

One of the major concerns in the management of transgenic crops is the risk of transgene flow from transgenic crops to conventional cultivars through pollen dispersal. Thus, accurate estimation of transgene flow is necessary to manage levels in non-GM crops. A number of field studies have been conducted in recent years to investigate gene flow through pollen dispersal (Jia et al., 2007; Kawashima et al., 2007; Klein et al., 2003; Rognli et al., 2000; Wang et al., 1997). These studies revealed that pollen transport and gene flow decreased with distance from the pollen source, depending upon wind speed and direction. Okubo and Levin (1989) summarized that the decreasing transport rate of dry air-borne spores or pollen with distance from a wide source could be fitted by an inverse power law or a negative exponential function, depending on the details of the case. Aylor et al. (2003) also reported that an inverse power law was relevant to fit the distribution of maize outcrossing rate. Measured

* Corresponding author: tushi@affrc.go.jp statistical information about cross-pollination rates between genetically modified (GM) crops and non-GM crops can be used to determine isolation distances required to prevent contamination by transgene flow and to formulate a policy for the coexistence of GM and nonGM crops (Messean et al., 2006).

However, recent studies on long-distance dispersal have shown that pollen dispersal from a point source follows a fat-tailed distribution that drops more slowly than a negative exponential function. Thus, those fitting functions cannot necessarily be extrapolated to longer distances because they were not derived from a physical or biological basis (Nathan et al., 2008). This implies that such fitting functions are not appropriate for estimation of pollen dispersal.

In estimating accurate transgene flow, predictions by numerical models are a better choice because they can be applied to any required set of conditions, such as wind variability, size of donor and recipient plots, topography, and biological effects. There are several mechanistic models employing measured wind speed and direction that can be used to calculate pollen transport. 


\section{T. Ushiyama et al.}

One of the most popular models is the Gaussian plume model (GPM), which computes the horizontal and vertical distribution of particles under the influence of diffusion and advection approximated as Gaussian distributions (Okubo and Levin, 1989). The GPM is simple but useful for grasping an overview of pollen dispersal (e.g., Loos et al., 2003; Yao et al., 2008). However, GPMs are limited to homogeneous landscapes because they are inherently unable to deal with spatial heterogeneity. Another popular mechanistic model that incorporates the effect of diffusion more precisely is the Lagrangian stochastic (LS) model (Aylor et al., 2006; Jarosz et al., 2004; Kuparinen, 2006; Kuparinen et al., 2007; Wilson and Sawford, 1996). The LS model deals with atmospheric turbulence explicitly in the calculation of particle dispersal by using wind speed data at a high time resolution. Therefore, the LS model is the best model for predicting long-distance dispersal where atmospheric turbulence plays an important role (Wilson and Sawford, 1996). However, the LS model also requires prescribed wind data.

Regional atmospheric models are able to predict wind field and other meteorological variables in spatial resolutions of several meters to tens of kilometers and in temporal resolutions of several seconds. Moreover, these models can take into account the dynamical effect of topography, inhomogeneous canopy distribution, and obstacles that cannot be incorporated into the two models mentioned above. Recently, regional models were used to predict alder or oak pollen dispersal within a range of a few hundred kilometers; these models showed advantages in producing realistic pollen dispersion by using a realistic predicted wind field (Helbig et al., 2004; Pasken and Pietrowicz, 2005; Schueler and Schlünzen, 2006). Dupont et al. (2006) predicted field-scale maize pollen dispersal by using a three-dimensional (3-D) atmospheric model and a diffusion transport model; field experimental data were reproduced as well. When compared with results from an LS model using statistically derived wind data, their data showed better reproduction owing to their use of a realistic wind field predicted by the atmospheric model. However, the predicted pollen ground deposition rates significantly differed from the observed amount.

In the present study, we conducted numerical experiments on maize pollen dispersal and cross-pollination by applying the 3-D regional and diffusion transport models A2Cflow and A2Ct\&d. A2Cflow predicts atmospheric state using an Eulerian approach, and A2Ct\&d predicts particle transport using a Lagrangian approach. For calculation of particle transport, the Lagrangian approach is believed to be more accurate because its advection equation is simpler and requires less approximation. However, the Lagrangian approach entails a large computational cost because a substantial number of particle trajectories must be calculated to obtain a smooth distribution. In the A2Cflow model, a kernel method is incorporated to reduce the computational cost of the Lagrangian approach. The details are described in the Materials and Methods section.

The pair of models used in this study was developed by Yamada Science and Art Inc. to simulate the turbulent transport of airborne materials based on the work of Yamada (1982) and Yamada and Bunker (1988). The models were tested against (a) nocturnal drainage flow in the California Geysers area (Yamada, 1981) with tree canopy effects (Yamada, 1982); (b) pollutant transport in Brush Creek, Colorado (Yamada and Bunker, 1988); (c) dispersion of airborne materials at Vandenberg Air Force Base, California (Yamada et al., 1992); and (d) tracer gas dispersion in southern Nevada to northwestern Arizona (Yamada, 2000). The approach provided satisfactory results in these instances. In the present study, we first predicted the wind field using the A2Cflow model and then calculated pollen dispersal by the A2Ct\&d model. Pollen dispersals from both the donor and recipient maize were calculated, and then the cross-pollination rates were calculated from the two distributions.

The most frequently used method to restrict pollen dispersal is to isolate the target plots from the GM plots by certain distances. Here, we propose another way to manage gene flow - by setting up windbreaks at certain locations. Comprehensive studies on the dynamical effects of windbreaks on natural wind profiles (e.g., Wang and Takle, 1995) have been reported. Generally, windbreaks suppress wind speed on their lee side (downstream side) within a distance of 10-20 times their height. The denser the windbreak, the narrower is the area of wind suppression. In the case of very dense windbreaks, such as a board, the air flow on the lee side circulates toward the upstream direction. Therefore, a moderate-density windbreak is more effective than a very dense windbreak to suppress wind speed on the lee side. Although the effects of wind reduction by windbreaks have been well studied, their effects in suppressing pollen dispersal and cross-pollination rates have not yet been reported. These effects were investigated in several experimental fields in Japan and will be described in the first part of this paper. The effects were then investigated using numerical experiments, and the computed variations in pollen dispersal and cross-pollination rates because of the various windbreaks will also be discussed.

\section{RESULTS}

\section{Field experiments}

Two kinds of maize cultivars (Zea mays L.) were raised in an experimental field. The experimental field contained 


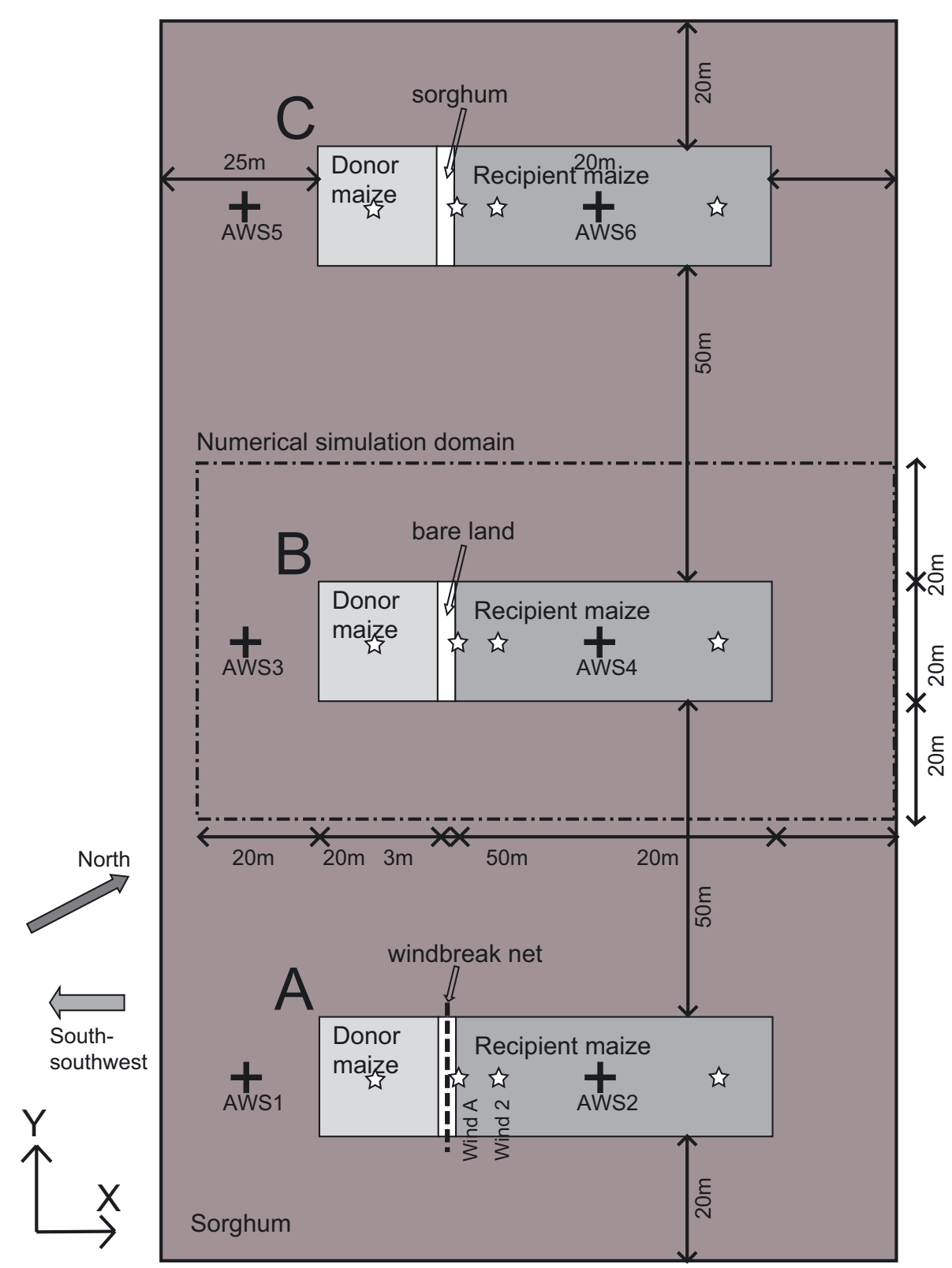

Figure 1. Arrangement of the experimental cornfield. Three pairs of donor and recipient maize plots were located in parallel; the remaining space in the cornfield was covered by sorghum, except between donor and recipient plots where a windbreak net (A), bare land (B), and sorghum (C) were positioned. Each experimental domain (pair of donor and recipient plots) had two automatic weather stations (AWSs, plus marks) and four pollen samplers and anemometers (open stars). A broken-dot line square in the center domain represents the arrangement of the numerical simulation domain.

three domains, each of which included a pair of donor and recipient plots with windbreaks in between, as illustrated in Figure 1. The windbreaks comprised a 6-m-tall windbreak net in domain $\mathrm{A}$, bare land in domain $\mathrm{B}$, and a sorghum windbreak in domain $\mathrm{C}$. The heights of the plants and windbreaks are summarized in Table 1. The donor maize in domain A grew higher than the others because the temperature around it was higher owing to the lighter wind resulting from the windbreak net.

Figure 2 shows the pollen number variations in the donor and recipient plots of each experimental domain. The numbers in the recipient areas in Figure 2 indicate the sampler averages, but samples that were statistically determined to be outliers were omitted. As shown in 


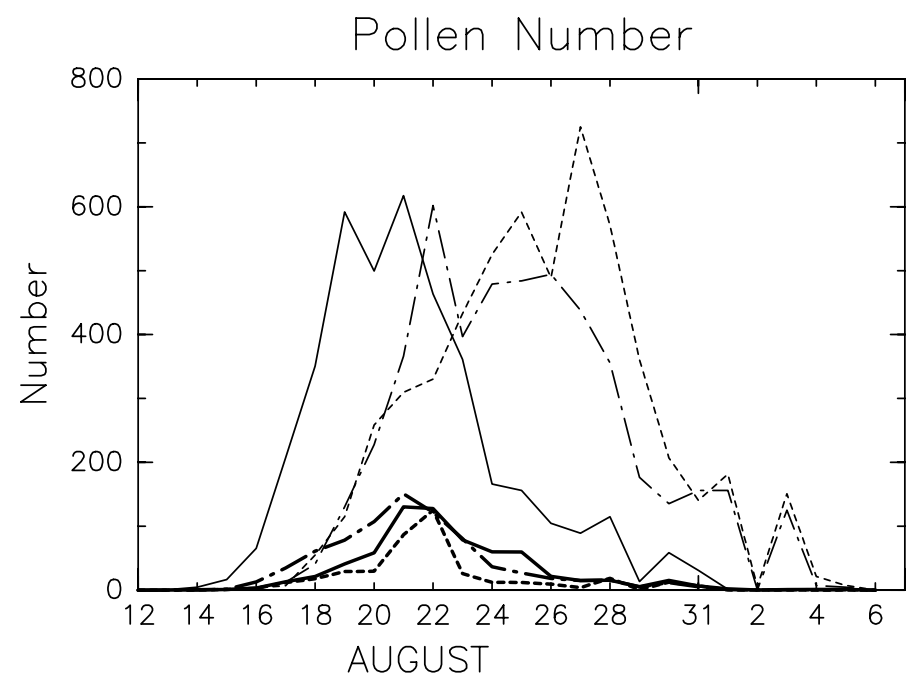

Figure 2. Variation of sampled pollen number. Three fine lines indicate the pollen number sampled in the donor plot, and three bold lines indicate that sampled in the recipient plot. Solid, broken, and broken-dot lines represent the A, B, and C experimental domains, respectively.

Table 1. List of plant heights $(\mathrm{cm})$.

\begin{tabular}{ccclc}
\hline & Donor maize & Recipient maize & Windbreaks in between & Buffer zone \\
\hline A & 189.3 & 130.5 & Net; 6 m high and 24 m wide & 124.3 \\
B & 167.3 & 130.5 & None & 124.3 \\
C & 160.2 & 118.3 & Sorghum; 182.7 cm tall & 124.3 \\
\hline
\end{tabular}

Figure 2, the anthesis of the plants was from Aug. 13, 2007 to Sep. 5, 2007. Most of the tassels started to disperse pollen on Aug. 17, except for the donors of domain A, which started two days earlier. Pollen dispersals were active from Aug. 18 to 29; maximum dispersals occurred from Aug. 21 to 22 in most of the domains, with the exception of the donor of domain $\mathrm{B}$, which reached maximum dispersal on Aug. 27.

Figure 3 shows the variation in the meteorological variables measured by an automatic weather station (AWS1). The temperature showed clear diurnal variations with several cooler days due to cloudy or rainy conditions (Aug. 18, 23, and 28-31). The wind speeds are displayed in $x(\mathrm{U})$ and $y(\mathrm{~V})$ components. Along with temperature, the rightward wind speed (positive $x$ direction in Fig. 1) also showed clear diurnal variation, blowing uphill in the daytime (recipient to donor direction) and downhill in the nighttime (donor to recipient direction), which is typical of valley and mountain breezes. Since the uphill wind prevailed in the daytime when the pollen emission rate was high, cross-pollination rarely occurred except on Aug. 21 and 22, when a downhill wind continued to blow throughout the entire day. Furthermore, since the donor pollen emissions were near the maximum on those dates in domains $\mathrm{A}$ and $\mathrm{C}$ and sufficiently large in domain B (Fig. 2), this period was the most effective for cross-pollination.

Figure 4 shows the observed cross-pollination rate averaged over six recipient plant ears at each of the 4-m sampling intervals from the donor boundary (see Materials and Methods section for details). Data from the donorside edge $(x=3 \mathrm{~m})$ are omitted because they became corrupt during data-sampling operations. All three lines show cross-pollination rate decreases from the donor source following an inverse power law, which is general pollen dispersal pattern reported by previous studies (e.g. Okubo and Levin, 1989). The cross-pollination rates in A and $\mathrm{C}$ appeared to be smaller than those in B within $25 \mathrm{~m}$ from the donor boundary, but the difference was not seen beyond $25 \mathrm{~m}$ from the donor boundary. However, statistical analysis of slopes and intercepts of two regression lines (fitted to inverse power law functions; Zar, 1984) showed that the cross-pollination rates of $\mathrm{A}$ and $\mathrm{C}$ were smaller than B when data at all distances were analyzed. These results confirmed that the net and plant windbreaks between the donor and recipient plots were effective in reducing pollen dispersal and cross-pollination.

Figure 5 shows the observed horizontal distributions of the cross-pollination rates. This figure amplifies the findings shown in Figure 4, i.e., that the cross-pollination 
Wind speed OBS/SIM A
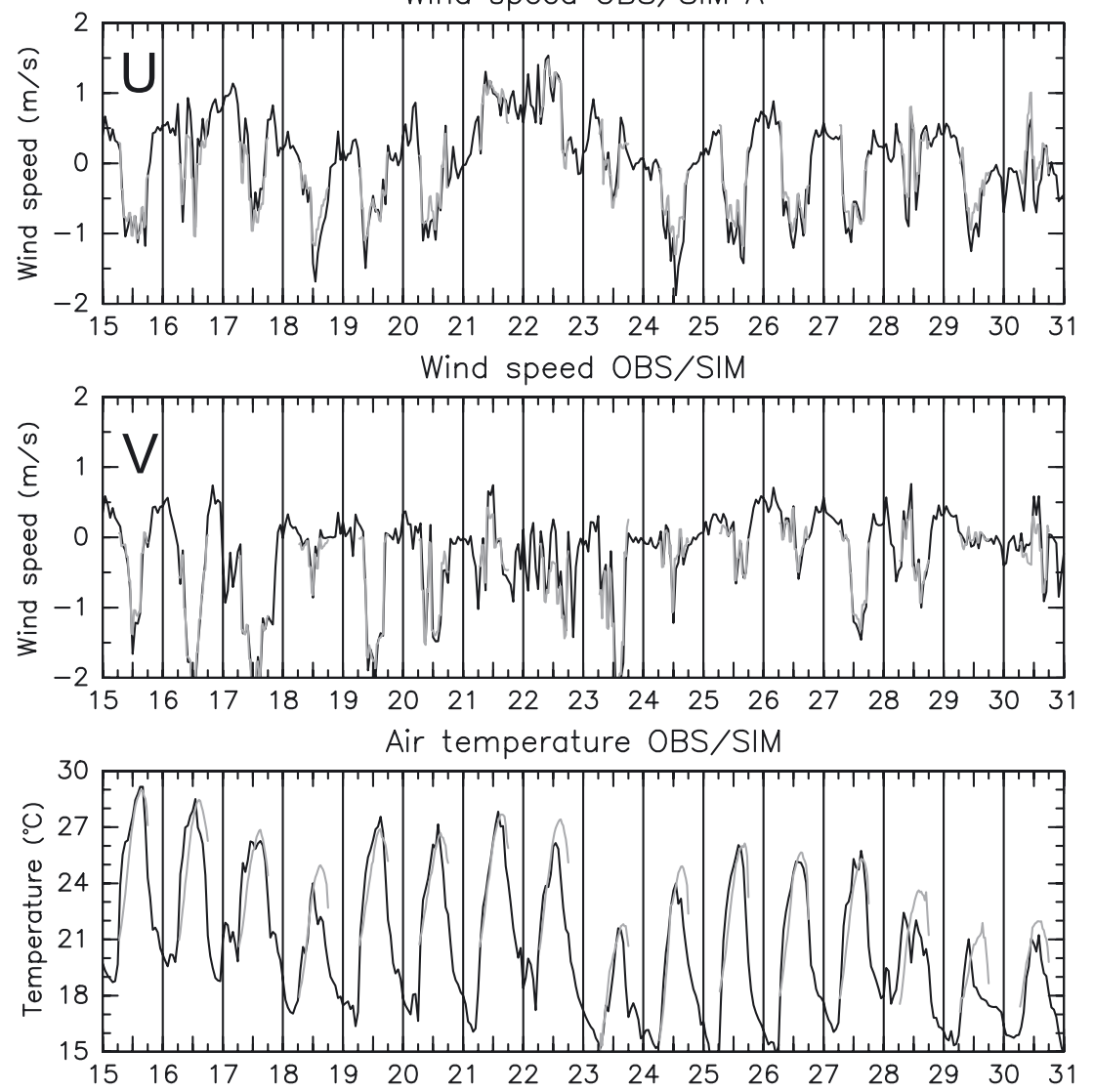

Figure 3. Time series of wind speed in $x(\mathrm{U})$ and $y(\mathrm{~V})$ components and air temperature $2.5 \mathrm{~m}$ above ground level in domain A. Black lines indicate the values measured by AWS, and gray lines are the predicted amounts.

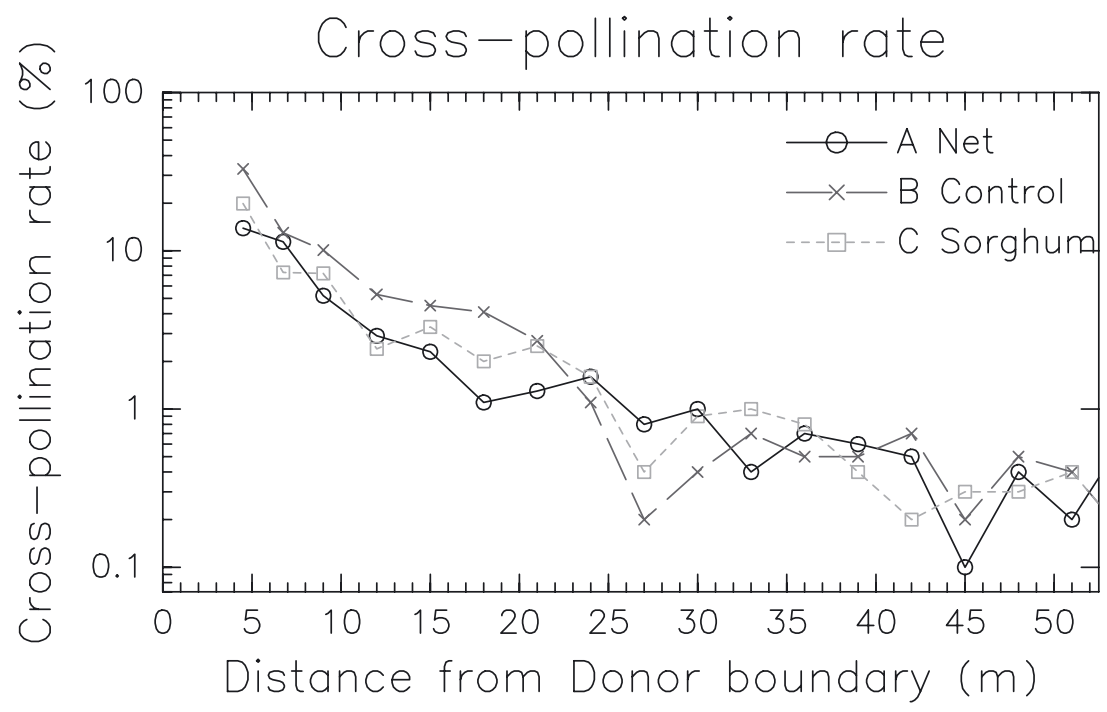

Figure 4. Observed cross-pollination rate in each experimental domain, A-C, averaged at the 4-m distance intervals from the donor boundary. Three types of lines and marks represent the results in the three domains as shown in the upper right corner, A: windbreak net, B: bare land, and C: sorghum. 


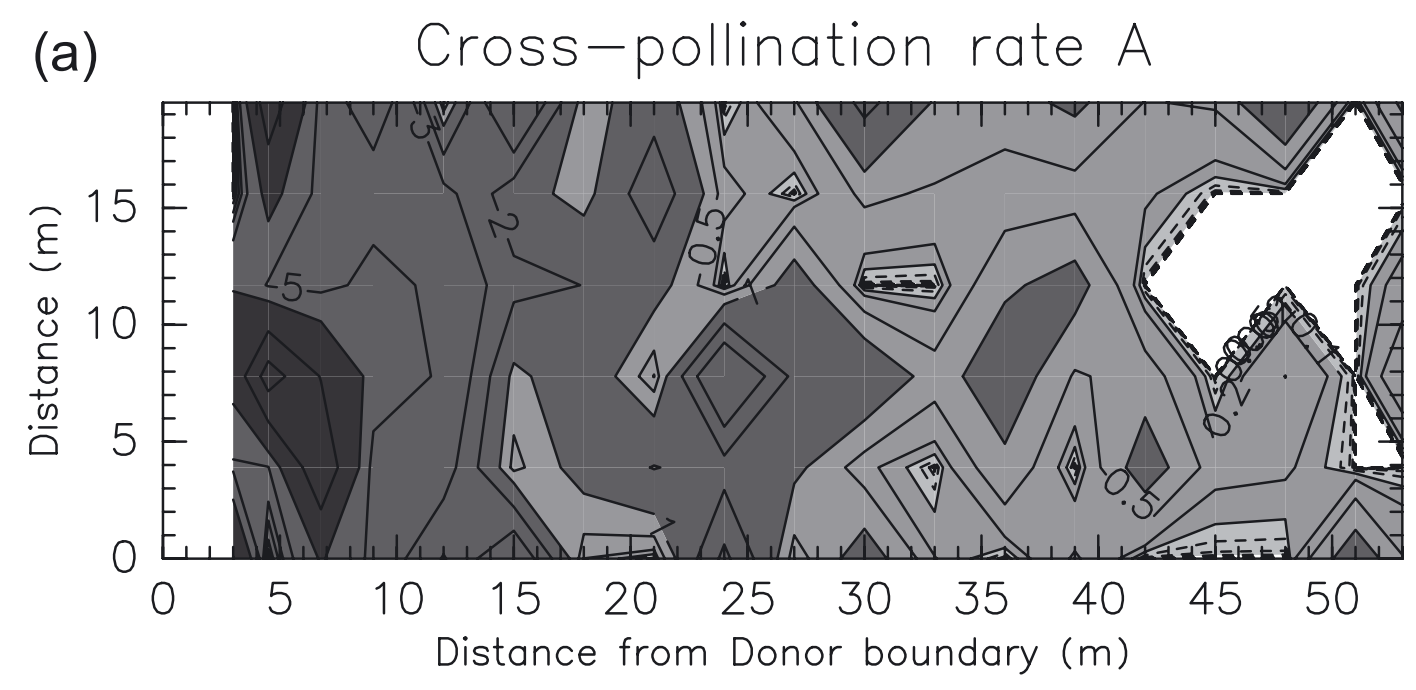

(b) Cross-pollination rate $B$

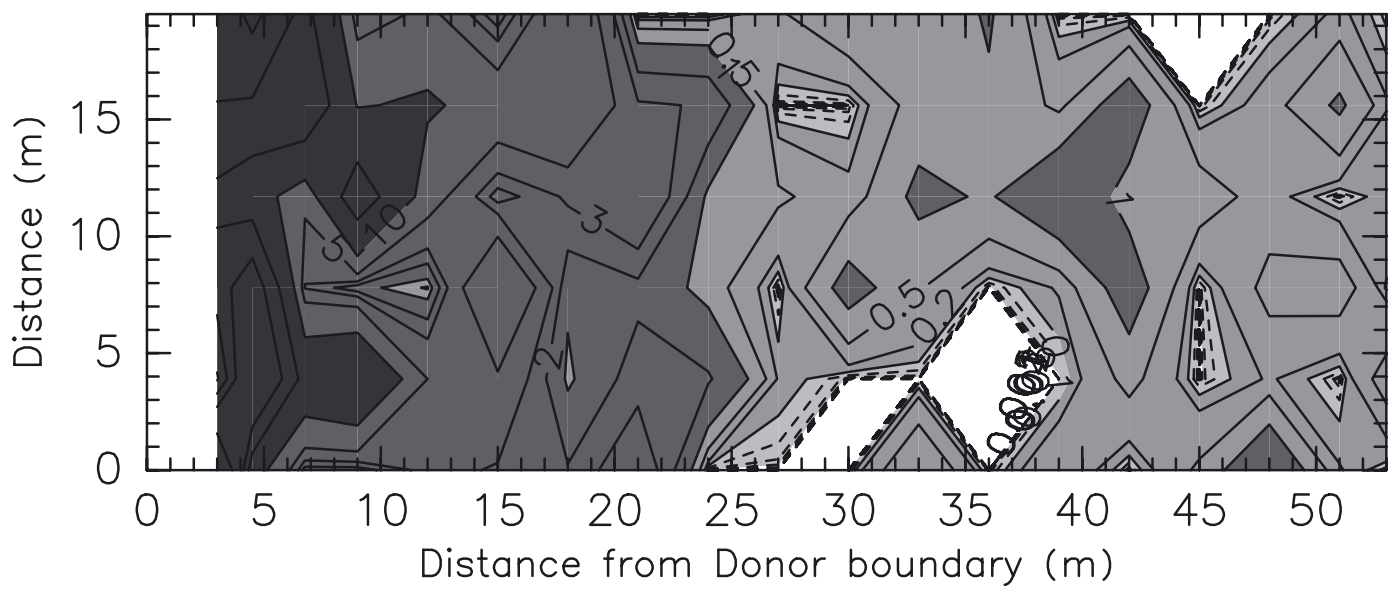

(c) Cross-pollination rate $\mathrm{C}$

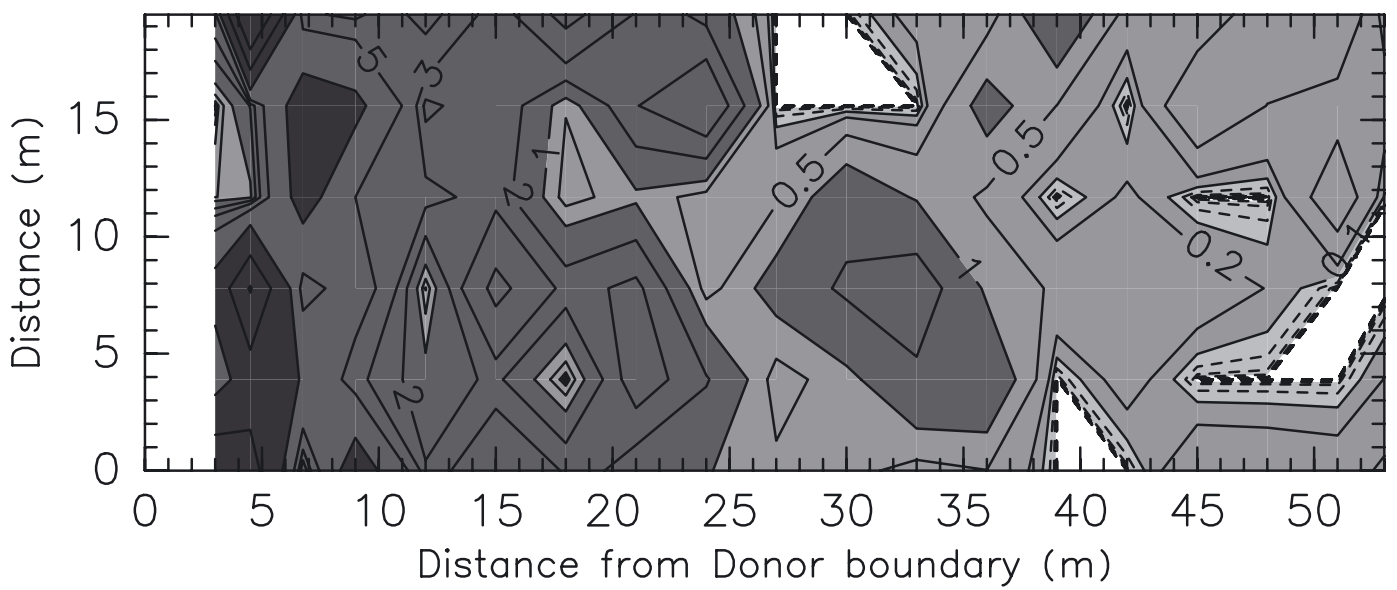

Figure 5. Horizontal distribution of the measured cross-pollination rates in the respective experimental domains (A-C). The color shadings are separated by $10,1,0.1$, and $0.01 \%$. 
rate near the donor boundary in domain B was larger than that in domains $\mathrm{A}$ and $\mathrm{C}$. This figure also exhibits a large variation in cross-pollination distribution in a range of three orders of magnitude. This deviation is most likely the result of the uneven development, growth and fertilization of the plants.

\section{Numerical experiments for reproduction of the measured cross-pollination}

We conducted a set of numerical experiments on the air flow field and pollen dispersal for the respective experimental domains $(\mathrm{A}-\mathrm{C})$ to reproduce the measured crosspollination rates described in the last subsection.

Figure 3 shows that the predicted wind speed just above the tassels was close to the measured wind speed in domain A. The differences between the predicted and measured wind speeds were at most $0.2 \mathrm{~m} . \mathrm{s}^{-1}$, but for most of the time the difference was zero. Since the observed wind speeds were incorporated into the model by nudging (or Newtonian relaxation (Stauffer and Seaman, 1990)), this result was a confirmation that the data nudging worked well. However, the temperature variation also agreed well even though it was not nudged. Prediction of correct temperature is important because temperature is associated with turbulence strength, which is related to the predicted wind speed, and in turn, the turbulent transport of pollen. The wind speeds and temperatures in domains $\mathrm{B}$ and $\mathrm{C}$ also showed good agreement with the model, as in domain A.

Figure 6 shows the predicted wind speed distributions with stream lines representative of each domain. The gray shadings in the figure represent the wind speed shown by the ratios relative to the wind speed at the upstream (left) boundary. The figure is a snap shot of a predicted vertical cross-section at the center in the $y$ direction at 12:00 LT Aug. 21, when winds in the donor to recipient direction prevailed and the pollen emissions were near their maximum. The various windbreaks between the donors and recipients, as in Figure 1, were located from 39 to $42 \mathrm{~m}$ in the $x$-dimension, with a windbreak net used in domain $\mathrm{A}$, bare land in domain $\mathrm{B}$, and a plant windbreak (sorghum) in domain $\mathrm{C}$.

In Figure 6a, the wind speed reduction was significant on the lee side of the windbreak net. A wind speed reduction of $30 \%$ extended for more than $15 \mathrm{~m}$ on the lee side and close to the height of the net $(6 \mathrm{~m})$. A wind speed reduction of $10 \%$ extended up to $30 \mathrm{~m}$ on the lee side near the ground and farther away at $5 \mathrm{~m}$ above the ground. A significant wind speed reduction was also found on the upstream side of the net. This reduced wind speed above the recipient plot could suppress pollen transport, while the reduced wind inside the donor plot could suppress the release of donor maize pollen, resulting in a smaller cross-pollination rate over the recipient plot. Strong vertical air motion was found in domain A; however, it had a secondary effect on subsequent total pollen transport. In Figure 6b, there was little wind speed variation except for a small area of wind speed acceleration between the donor and recipient areas. In domain B, donor pollen was transported over the recipient area without wind speed loss. In Figure 6c, a small but significant area of wind speed reduction was found within the donor plot and lee side of the windbreak. This wind speed reduction could reduce donor pollen transport in domain $\mathrm{C}$. Vertical air motion was also found over the plant windbreak but was significantly weaker than that in domain A. The ability to visualize air motion, as in Figure 6, is an advantage of numerical predictions since it is hard to observe in a real field. Such visualization would be useful for verifying the dynamical effects of windbreaks and the process by which they reduce pollen transport.

Figure 7 shows the horizontal distributions of the predicted cross-pollination rates. They show smoother distributions but similar characteristics to those of field measurements in Figure 5; i.e., the cross-pollination rates were larger near the donor boundary and decreased away from the donor boundary, and the values of domain B were larger than those of domains A and C. Since this numerical prediction did not take into account the effect of inhomogeneity on plant growth and fertilization, the difference between the smooth distributions in the predictions and the rough distribution in the field measurements could be caused mainly by uneven plant growth and fertilization.

Figure 8 compares the predicted and observed crosspollination rates averaged at the sample distances from the donor boundary. This figure clearly shows the characteristics of the suppressing effects on the cross-pollination rate by windbreaks. The windbreak net in domain A suppressed the cross-pollination rate most effectively, and the plant windbreak in domain $\mathrm{C}$ was the next effective, compared to the largest cross-pollination rate in the control experiment in domain B. This feature was evident at all distances from the donor boundary in Figure 8 but was not recognized beyond $25 \mathrm{~m}$ in field measurements. The predicted cross-pollination rate in domains $\mathrm{B}$ and $\mathrm{C}$ were in good agreement with the measurements when slopes and intercepts of both regression lines were statistically examined (Zar, 1984). However, the predication for domain A underestimated the measured cross-pollination rate beyond $25 \mathrm{~m}$ from the donor boundary.

Before discussing the inconsistency of predicted and measured cross-pollination rate in domain $\mathrm{A}$, predicted wind speed around the windbreak net was verified by comparing with measured wind speed which was not included in the assimilation. Figure 9 shows that the predicted wind speed agreed well with the measured wind 


\section{T. Ushiyama et al.}
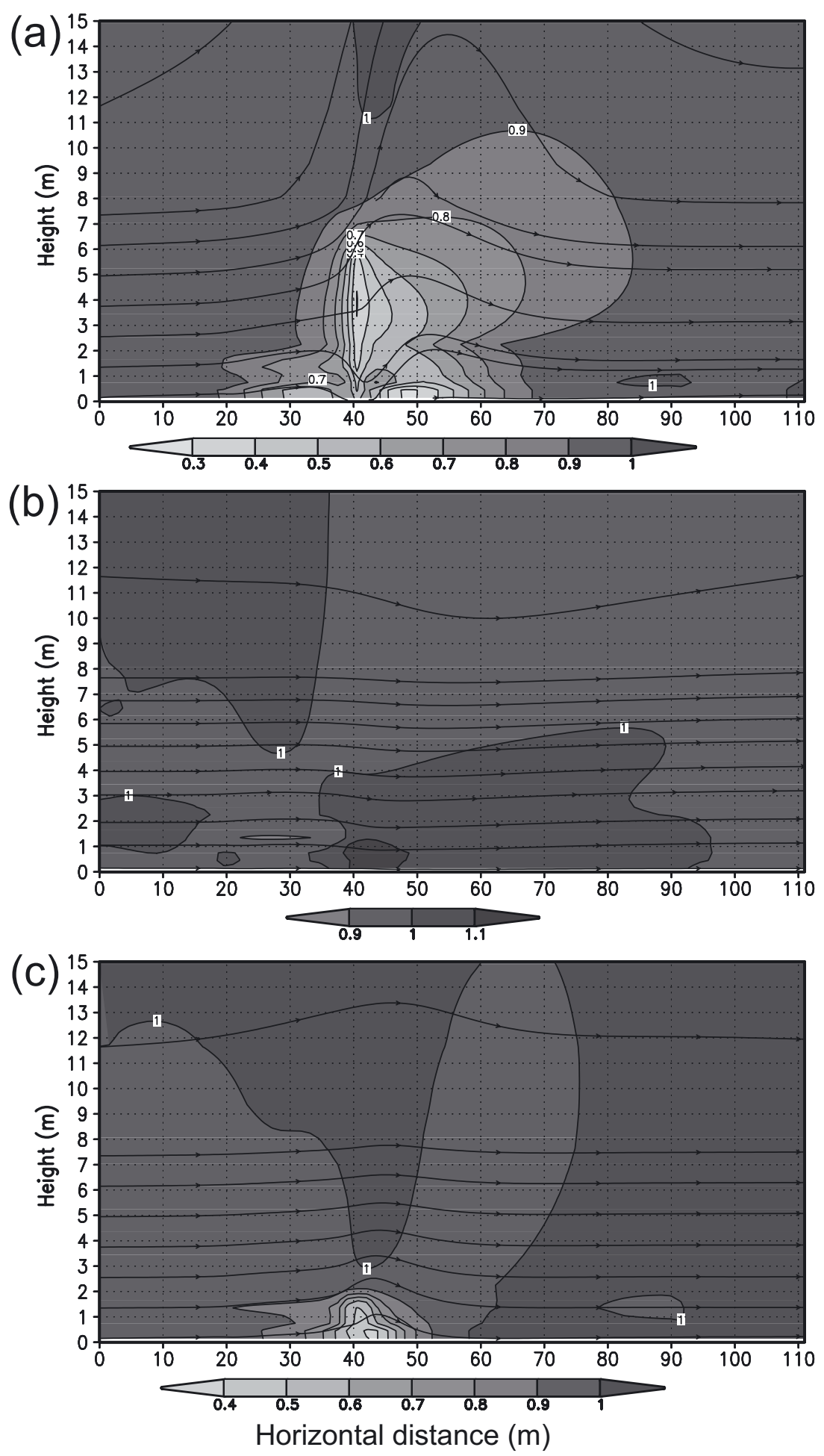

Figure 6. Vertical cross-section of predicted horizontal wind speed relative to the upstream boundary. The cross-section was along the center of the cornfields for domains A-C at 12:00 on Aug. 21. Stream lines indicate air flow, and vertical wind speed was enhanced 10 -fold. 

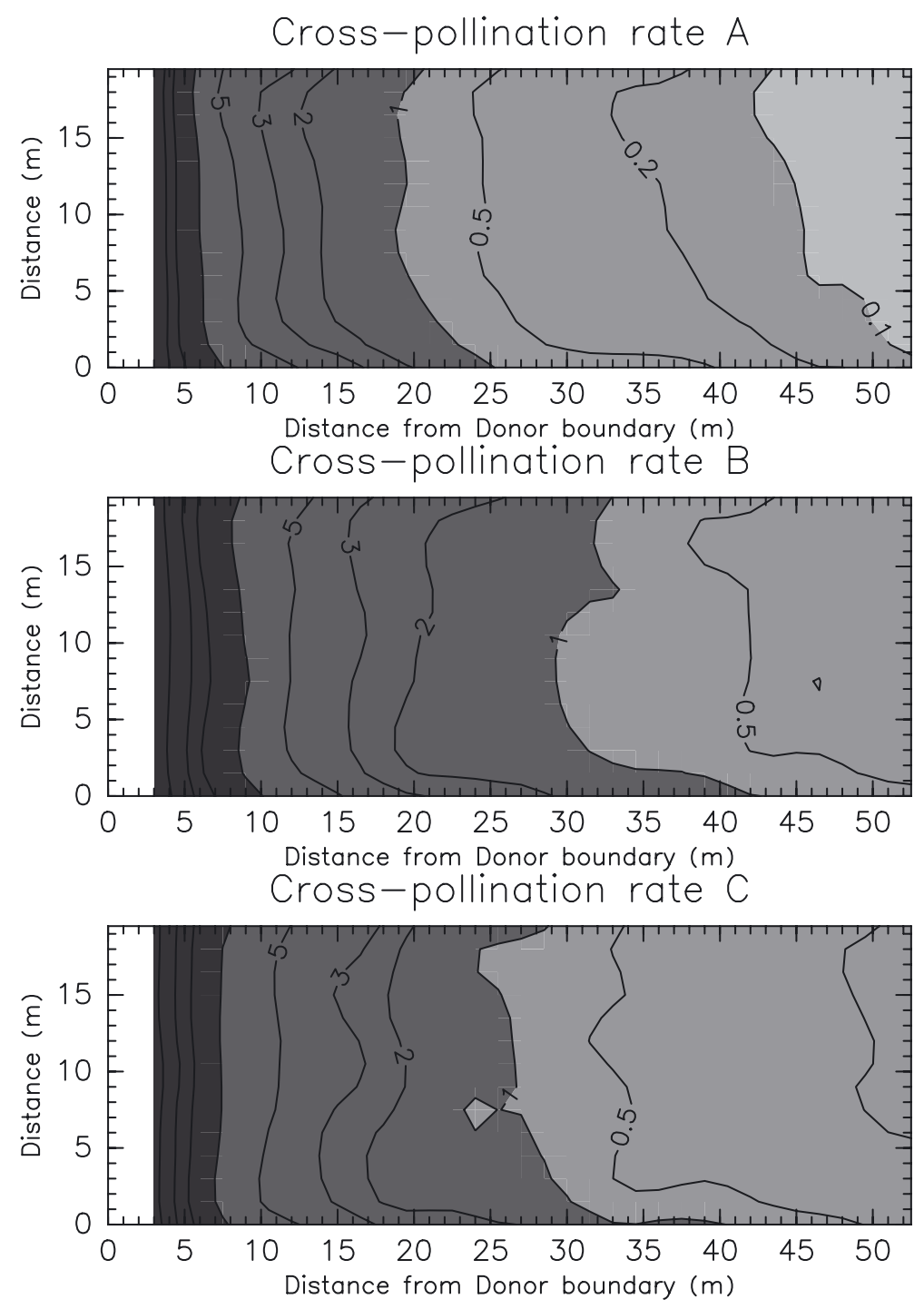

Figure 7. Same as Figure 5 except for predicted cross-pollination rate.

speed during Aug. 21 and 22 when the bulk of crosspollination occurred. This implies that the model performed quite well with regard to wind speed suppression by the windbreak net at the wind-nudging height. Thus, the model performance on the windbreak net was not the cause of the inconsistency.

There are two possible reasons for the underestimation of predicted cross-pollination rate in domain A. First, wind speed above the wind-nudging height $(2.5 \mathrm{~m})$ might have been underestimated due to a smaller wind speed in the assimilated GPV (Grid Point Value data provided by Japan Meteorological Agency) than that measured by AWS. Since the windbreak net was higher than the plant windbreak in domain $\mathrm{C}$, the wind reduction effect might have been enhanced more than in the plant windbreak case (Fig. 6). However, since there were no observed wind data except at the 2.5 -m height, we could not compare the predicted and observed effects on wind speed reduction above the $2.5-\mathrm{m}$ height.

Second, the uneven growth of the recipient maize might have caused large deviations in the observed cross-pollination rate. The variation in the measured cross-pollination rate was quite large (Fig. 5) because many factors, such as wind, turbulence, plant growth, pollen emission rate, and fertilization efficiency, affect final cross-pollination rates. Further, the predicted crosspollination rate was within a standard deviation of the measured amount (not shown). Nevertheless, this does not explain why results from domains $\mathrm{B}$ and $\mathrm{C}$ were in good agreement with the model, while A was not. 
T. Ushiyama et al.

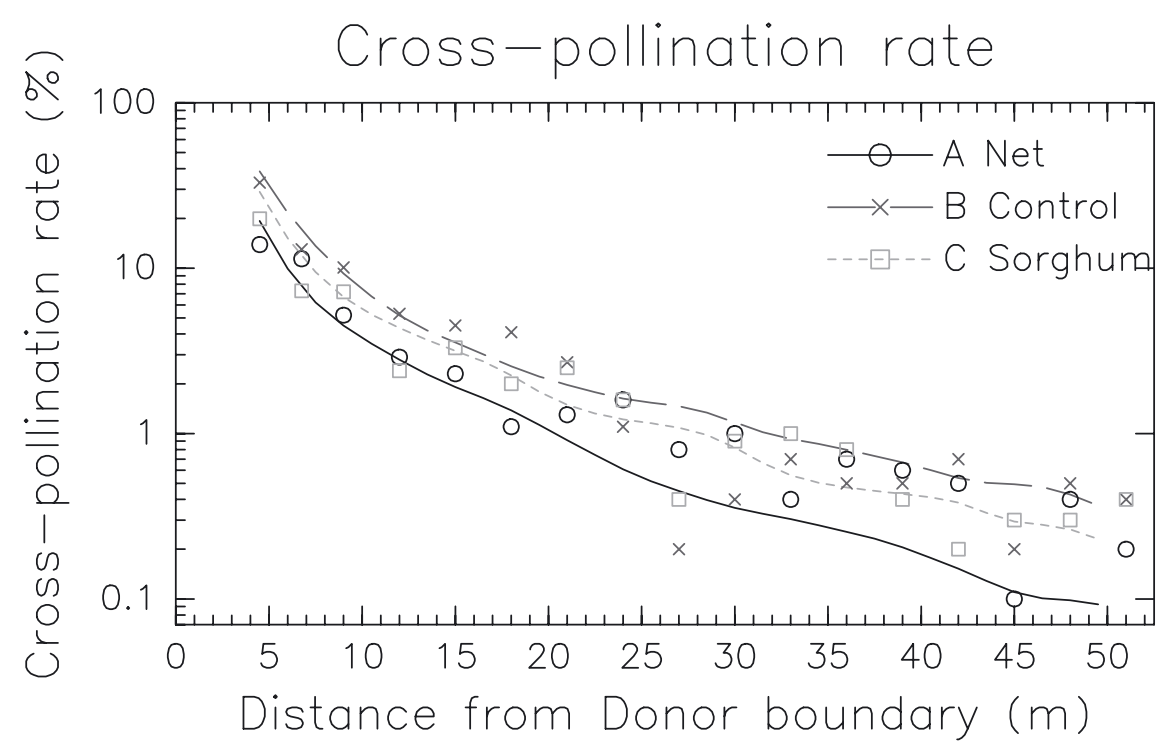

Figure 8. Predicted cross-pollination rate in each experimental domain, A-C, averaged in the $y$ direction. The three types of lines indicate the predicted amounts. The circular, cross, and square marks indicate the measured amounts, just as in Figure 4.
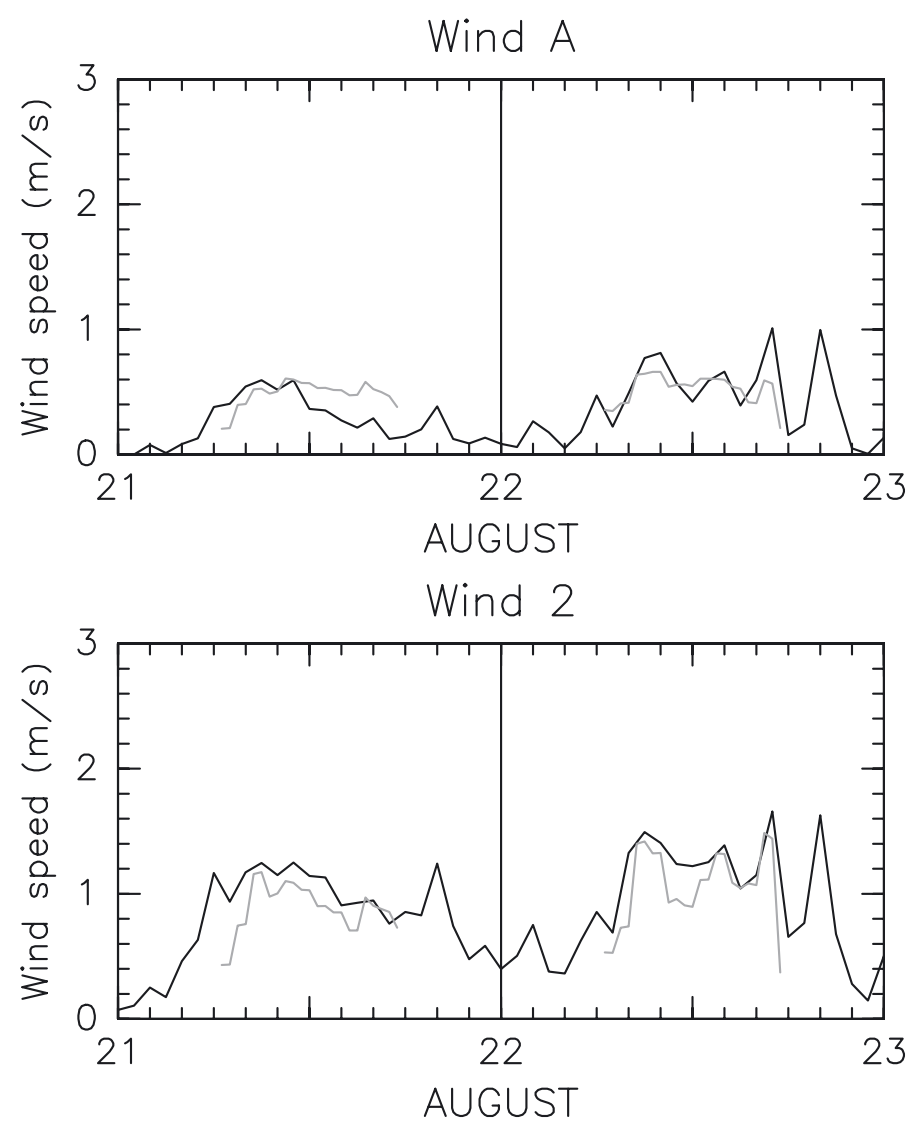

Figure 9. Observed (black) and predicted (grey) wind speed around the windbreak net in domain A. Observed locations are shown by open stars in Figure 1 (wind A and wind 2). Upper panel shows the wind speed nearest the windbreak net, and the lower panel shows the data recorded downstream of the former location. 
Prediction of maize pollen dispersal

Table 2. Suppression effect of cross-pollination rate in the lee side of windbreaks.

\begin{tabular}{llcc}
\hline & Windbreak & Average cross-pollination rate & Isolation distance for 0.9\% \\
\hline A & 6-m-tall 1-mm-mesh windbreak net & $39 \%$ & $21 \mathrm{~m}$ \\
B & Nothing & $100 \%$ & $33 \mathrm{~m}$ \\
C & 182.7 cm tall sorghum windbreak & $70 \%$ & $27 \mathrm{~m}$ \\
Ex1 & 3-m-tall 1-mm-mesh windbreak net & $50 \%$ & $24 \mathrm{~m}$ \\
Ex2 & 3-m-tall 2-mm-mesh windbreak net & $61 \%$ & $27 \mathrm{~m}$ \\
\hline
\end{tabular}

Further analysis will be required to examine the validity of the model in reproducing the suppressing effects of windbreak nets on cross-pollination rate. Measurements of wind speed profile at least up to the height of windbreak net will be desirable to examine wind speeds in the lee side of windbreak nets. In this study, the numerical model was validated for reproducing the suppressing effects on cross-pollination rates in the plant windbreak and no windbreak cases.

As shown in Figure 8, windbreak net and plant windbreak suppressed cross-pollination rate in the lee side. Suppression rates averaged for the distance from 4.5 to $51 \mathrm{~m}$ by windbreaks are summarized in Table 2 . The suppression rates by the windbreak net and by the plant windbreak were approximately $60 \%$ and $30 \%$, respectively. The suppression rates of the windbreak net and the plant windbreak increased with distance (from $4.5 \mathrm{~m}$ to $51 \mathrm{~m}$ ) from 45 to $75 \%$ and from 20 to $40 \%$, respectively. When we consider the $0.9 \%$ cross-pollination requirement, the no windbreak case requires an isolation distance of $33 \mathrm{~m}$ between the donor and recipient plots. On the other hand, in the cases of the windbreak net and the plant windbreak isolation distances of $21 \mathrm{~m}$ and $27 \mathrm{~m}$ were required respectively. However, the results in crosspollination rates included effects of variable pollen emission rate, especially in their donor plots and thus did not entirely represent the effects of the windbreaks.

\section{Numerical experiments for idealized windbreak nets}

The numerical model was utilized to investigate the effects of various windbreak nets on suppressing crosspollination rates. Although the model had shortcomings in reproducing cross-pollination rates beyond $25 \mathrm{~m}$ from the donor pollen source, the model was able to compare cross-pollination rates between windbreaks. Since the 6-m-tall windbreak nets were costly to install in the field, modeling of windbreaks that are much easier to install, such as half-height windbreak nets $(3 \mathrm{~m})$ with $1-\mathrm{mm}$ and 2-mm-mesh, should be verified. In the case of the 2-mm-mesh net, the drag coefficient and leaf area density were determined from the wind tunnel measurements, as was done with the 1 -mm-mesh net. The horizontal arrangements and boundary conditions of the two additional experiments were exactly the same as those used in the domain A experiment.

Figure 10 exhibits the wind speed distributions for the half-height windbreak net experiments, similar to those shown in Figure 6. Figure 10a shows that the 3-m-tall, 1 -mm-mesh net reduced wind speed significantly, but the extent of $10 \%$ wind speed reduction on the lee side was about half that of the 6-m-tall net (Fig. 6a). However, on the upstream side, the extent of $10 \%$ wind reduction was the same as that of the 6-m-tall net. Figure 10b shows that the extent of $10 \%$ wind reduction by the $3-\mathrm{m}$-tall, 2-mm-mesh net was much narrower on the lee side, but that wind reduction extended over the whole donor plot on the upstream side of the net.

Figure 11 compares the average cross-pollination rate for the original domain A (6-m-tall, 1-mm-mesh) and the two additional experiments. The suppression of the cross-pollination rate was reduced $24 \%$ on average when the height of the windbreak net was decreased from $6 \mathrm{~m}$ to $3 \mathrm{~m}$. The suppression effect of the $2-\mathrm{mm}$-mesh 3 -m-tall windbreak net was reduced $40 \%$ in average compared to the 1-mm-mesh 6-m-tall windbreak net. Crosspollination suppression levels by the 3-m-tall $1-\mathrm{mm}$ mesh and 2-mm-mesh windbreak nets were summarized in Table 2, and were $50 \%$ and $61 \%$, respectively. It implies that the 3-m-tall 2-mm-mesh windbreak net still suppressed the cross-pollination rate more than the plant windbreak of domain $\mathrm{C}$. This experiment suggests that a shorter and coarser windbreak net had a greater suppression effect on the cross-pollination rate than was expected from a smaller lee-side wind reduction. When we consider $0.9 \%$ cross-pollination threshold, 3-m-tall 1-mm-mesh and 3-m-tall 2-mm-mesh windbreak nets needed isolation distances of $24 \mathrm{~m}$ and $27 \mathrm{~m}$ between the donor and recipient plots, respectively. These isolation distances were larger than those of the 6-m-tall 1 -mm-mesh windbreak net $(21 \mathrm{~m})$, but smaller than the no windbreak case $(33 \mathrm{~m})$.

\section{DISCUSSION}

\section{Model validation}

In this study, pollen dispersal and cross-pollination rates between two adjacent maize cultivar plots were 
T. Ushiyama et al.
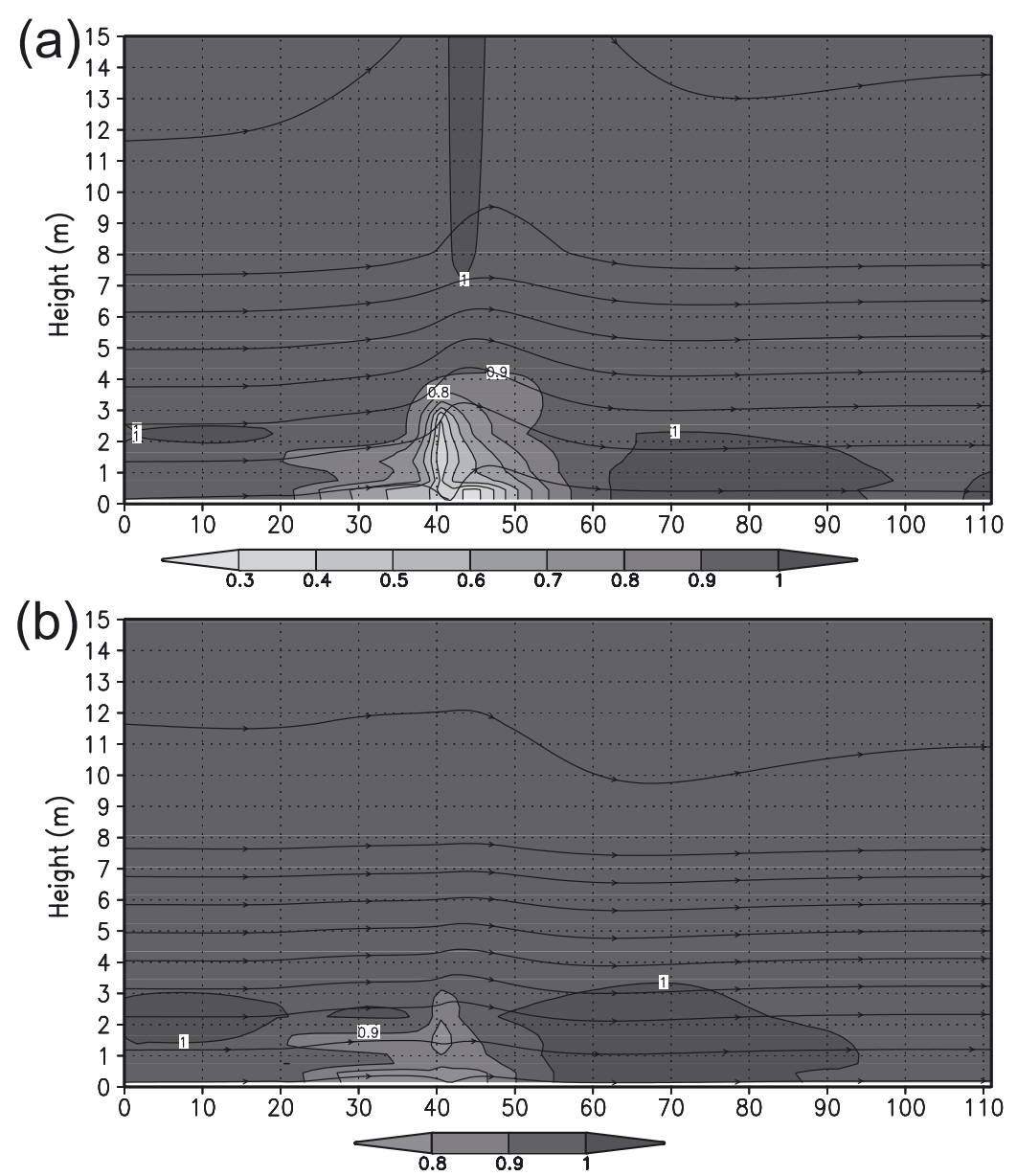

Figure 10. Same as Figure 6 except for the modified domain A. (a) 3-m-tall, 1-mm-mesh net and (b) 3-m-tall, 2-mm-mesh net.

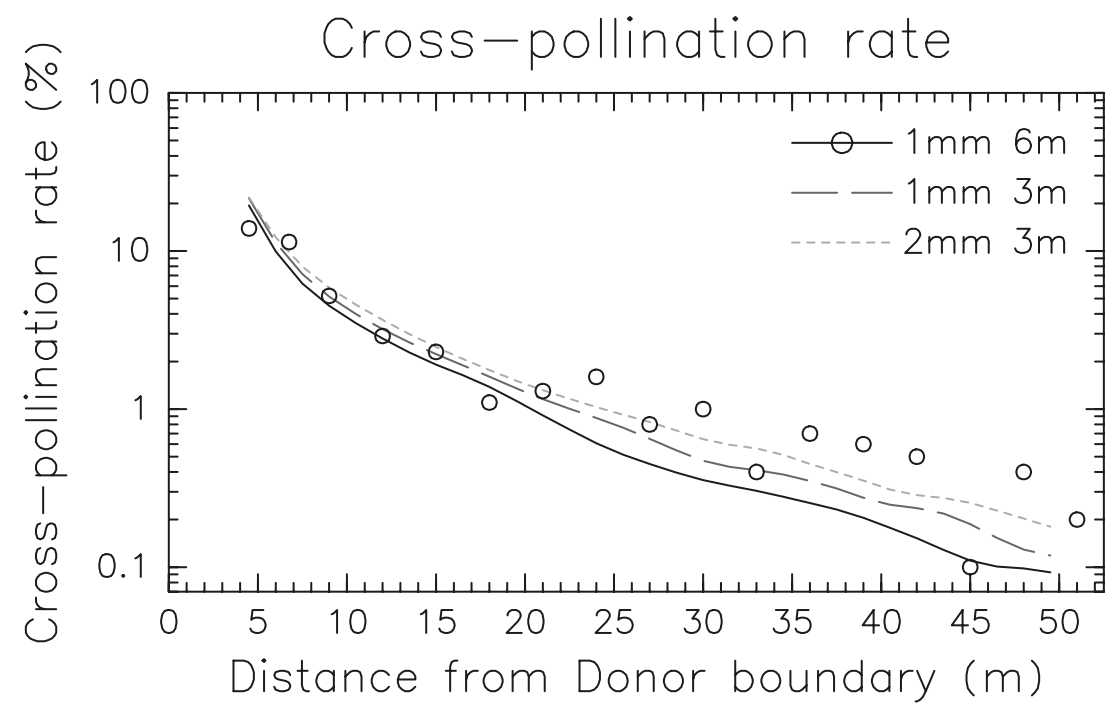

Figure 11. Same as Figure 8 except for the predicted cross-pollination rate of domain A, and domain A with a modified windbreak net for 3-m-tall, 1-mm-mesh net and for 3-m-tall, 2-mm-mesh net. 
investigated by a field experiment and numerical predictions by using a 3-D atmospheric model and a diffusion transport model. The resulting model reproduced the cross-pollination rate quite well in cases where plots had a plant windbreak or no windbreaks. However, the model underestimated the cross-pollination rate beyond $25 \mathrm{~m}$ from the donor boundary in plots using a windbreak net. The reason of underestimated cross-pollination rate in case of 6-m-tall windbreak net was most likely underestimation of wind speed over $2.5 \mathrm{~m}$ height where in situ wind measurements were made, and uneven plant growth or fertilization would be the second reason.

The numerical prediction of pollen dispersal and cross-pollination in this study has several advantages. First, this model reproduced the measured average crosspollination rate quite well in the control experiment (without windbreak) and the plant windbreak experiment. This is important because numerical studies of field-scale pollen dispersal involving such atmospheric models are quite limited. The only such study in our knowledge was Dupont et al. (2006). Since an atmospheric model can predict the wind field under any required set of conditions, such as topography, land use and presence of windbreaks, it is a powerful tool for pollen flow assessments.

Dupont et al. (2006) predicted pollen dispersal on the field-scale by using a 3-D atmospheric model and compared the results with field measurement data. They calculated pollen dispersal with a somewhat different method by using a diffusion-advection conservation equation, which is an Eulerian approach rather than the Lagrangian approach used in this study. They discussed (donor) pollen dispersal whereas we focused on crosspollination rate. Therefore, the results of the two studies are not directly comparable because the cross-pollination rate includes the pollen distribution of both donor and recipient sources. However, the accuracy of numerical prediction in this study is comparable to theirs: Agreements with the observed amounts were within about $100 \%$ in most of data points of ours and theirs. This implies that the model in the present study maintained good accuracy.

The second advantage of our model is that it can predict cross-pollination rates in addition to pollen dispersal. Most recent studies investigating the accurate calculation of pollen dispersal dealt with pollen dispersal only and were not concerned with the cross-pollination rate (Aylor et al., 2006; Dupont et al., 2006; Jarosz et al., 2004). This is reasonable, because prediction of cross-pollination includes further complex factors, such as recipient pollen distributions, the condition of ovules, and the time lag of silk growth (protandry), in addition to donor pollen dispersal. However, the present study reproduced the crosspollination distribution of maize plots fairly well. This implies that the model is valid for the assessment of maize gene flow at the field scale. It also confirmed that the assumptions used in this study were reasonable: (1) Deposition and resuspension of pollen on the plants were ignored (Dupont et al., 2006; Klein et al., 2003); (2) the cross-pollination rate was computed from the ratio of donor and recipient pollen concentration near the ears; (3) the instantaneous pollen emission rate was determined from the time derivative of air temperature near the ears (Kawashima et al., 2004), but the total count for the day was later adjusted to the in situ measurement; and (4) the protandry score was computed from the combination of drought stress and plant density (Angevin et al., 2008).

On the other hand, the model could not accurately reproduce the cross-pollination rate beyond $25 \mathrm{~m}$ from the donor boundary in the case of the windbreak net. This is the first study of numerical prediction of pollen dispersal and cross-pollination in which the effect of a windbreak net was incorporated. The drag force of the net was measured in a wind tunnel (Ushiyama et al., 2009) and appropriately incorporated into the model. As a result, the wind speeds close to the net on the downstream side agreed well with observations at the wind-nudging height. However, the wind speeds over the wind-nudging (assimilation) height might not be realistic because assimilated GPV data might not represent such field scale wind speeds. This is a limitation of the prediction procedure in this study. We conclude that the wind speed profile above the windbreaks should be more carefully treated when the model includes windbreaks higher than the height of the assimilated wind measurements. Further experimental and numerical predictions are required to examine the validity of the model for windbreak effects in suppressing pollen dispersal and cross-pollination.

\section{Suppression of pollen dispersal and cross-pollination by windbreaks}

The numerical experiments demonstrated that the windbreak net and plant windbreak suppressed pollen dispersal and cross-pollination rates. This result was shown more clearly in the numerical experiments than in the field experiments. The field experiments shown in Figure 4 were pollinated beyond $25 \mathrm{~m}$ from the donor boundary, but because the absolute values of cross-pollination rates were so small, it is more likely that the recipient plots were contaminated by a few plants. However, the relationship between cross-pollination rates of the three experimental domains was consistent between the field and numerical experiments; the windbreak net was the most effective for pollination suppression, followed by the plant windbreak.

It should be noted that the cross-pollination rates shown in Figure 8 did not always represent the quantitative relationship between the suppression effects of windbreaks. During the period of dominant cross-pollination 


\section{T. Ushiyama et al.}

(Aug. 21 and 22), the recipient pollen emissions were mostly the same in the three domains, while the donor pollen emissions were not the same (Fig. 2). The donor pollen emission in domain B (control experiment) was less than in the others, which implies that the crosspollination rate in domain B was underestimated. From this fact, the suppression effect on cross-pollination by the plant windbreak would have been more pronounced if all three domains had constant donor pollen emissions compared to the result shown in Figure 8.

In addition to the two types of windbreaks used in the field experiment, suppression effects of another two kinds of windbreak nets were predicted: 3-m-tall, 1-mmmesh and 3-m-tall, 2-mm-mesh. These predictions revealed that a higher and denser windbreak net was more effective in suppression, but that lower and coarser windbreak nets still had substantial suppression effects.

The predicted wind speed distributions around the various windbreaks showed massive differences in their suppression of lee-side wind speeds (Figs. 6 and 10), while their suppression of cross-pollination showed rather moderate differences between the windbreaks (Figs. 8 and 11). This suggests that lee-side wind speed is not strongly influential on cross-pollination rate. However, wind speeds on the upstream side of the windbreaks were suppressed significantly in all the windbreak cases that showed significant suppression of cross-pollination rates. This suggests that upstream wind speed is an important factor in cross-pollination rate. It is important to suppress wind speed in the donor plot rather than in the recipient plot in order to reduce cross-pollination. The mechanism of suppressing cross-pollination by windbreaks could be described as "suppressing pollen dispersal from the donor plot by reducing wind speed in the donor plot". Based on this reasoning, we should be able to reduce cross-pollination more effectively by arranging the windbreak location to reduce wind speed in the donor plot. This experiment will be reported in a future paper.

These numerical experiments suggested that the model used in this study would be helpful when designing the isolation of a maize field to manage cross-pollination. The cross-pollination level could be estimated quantitatively by numerical prediction using the model. Since installation of windbreak nets incurs large costs, they are not practical for use in large commercial fields. In such cases, a plant windbreak would be more practical for suppressing cross-pollination rates. We plan to conduct an investigation of suppression of cross-pollination by plant windbreaks in a future study.

The model could be used for species other than Z. mays L. by modifying the pollen-fall speed. The model can also be applied to the assessment of pollen flow in various types of crops and should be a useful tool for assessment of transgene flow frequencies.

\section{MATERIALS AND METHODS}

\section{Field experiment}

Two kinds of maize cultivars (Z. mays L.) were raised in an experimental field at the Tsumagoi Station of the National Center for Seeds and Seedlings, Gunma, Japan $\left(36^{\circ} 27^{\prime} \mathrm{N}, 138^{\circ} 27^{\prime} \mathrm{E}\right.$, altitude $\left.=1300 \mathrm{~m}\right)$ in 2007 to study maize pollen dispersal and cross-pollination. The experimental field had three domains, each of which included a pair of donor and recipient plots. Windbreaks were placed between the donor and recipient plots to examine their suppressing effects on pollen dispersal and cross-pollination as illustrated in Figure 1. This experimental field was located on a mountain slope, and the donor plot was on the upslope side of the recipient plot. The upslope edge (left edge in Fig. 1) was approximately $20 \mathrm{~m}$ higher than the down-slope side. We could not eliminate the possibility of the slope's effect on donor pollen dispersal. However, the results of the numerical prediction showed good agreement with the observations in domains B and C. Therefore, the effect of the slope was judged to be negligible. The donor maize occupied an area of $20 \mathrm{~m} \times 20 \mathrm{~m}$, whereas the recipient maize occupied an area of $50 \mathrm{~m} \times 20 \mathrm{~m}$ (Fig. 1). The three domains, A-C, lay in parallel at 50-m intervals. Slightly shorter sorghum was planted around the maize plots, except in the windbreak area between the donor and the recipient maize pairs. The heights of the plants are summarized in Table 1. Domain A had a 6-m-tall and a 24-m-wide windbreak net with a 1-mm-mesh between the donor and recipient plots. Domain B had bare land in between and served as a control. Domain $\mathrm{C}$ had a plant windbreak (sorghum) that was slightly taller than the donor maize.

As shown in Figure 1, four pollen samplers, four anemometers, and two AWSs were placed in each experimental domain. One pollen sampler was in the donor area and the other three were in the recipient area. The samplers were the Durham type, and the data-collection time interval was 1 day. The AWS measured the wind speed, wind direction, temperature, relative humidity, and rainfall every hour at a height of $2.5 \mathrm{~m}$ above ground level on the uphill side (AWS1, 3, 5) and in the recipient area (AWS2, 4, 6) in each experimental domain, as shown in Figure 1. The anemometers measured wind speeds at $2.5 \mathrm{~m}$ above ground.

The donor maize was a yellow grain cultivar (dominant trait), "honey bantam", and the recipient maize was a white grain cultivar (recessive trait), "silver honey bantam". This would enable us to easily distinguish intracultivar-pollinated and intercultivar-pollinated grains through the xenia phenomenon. The xenia phenomenon usually refers to a situation in which the genotype of the pollen donor influences the maternal tissue of the fruit so as to produce a phenotypically demonstrable effect upon 
the seed grains of the recipient. In this experiment, when the ovules of the silver honey bantam were fertilized with the pollen of the honey bantam, the grains obtained from the silver honey bantam appeared rather yellow, thereby displaying a xenia effect. By counting the grains showing xenia among the grains collected from the silver honey bantam, we could easily estimate the intercultivar pollination rate between the two maize cultivars.

In maize, the silks are pollinated by pollen from other individual plants. Therefore, the term "intercultivar pollination" is correct when referring to pollination by a different cultivar species. In this paper, we refer to intercultivar pollination as "cross-pollination" and intracultivar pollination as "self-pollination".

In the field experiments, the maize was planted at 25 -cm intervals along rows $75 \mathrm{~cm}$ apart ( $y$-axis in Fig. 1); the rows ran across the shorter dimension of each plot. The cross-pollination rates were obtained by counting seeds showing and not showing xenia from an ear on the recipient maize plants. The sampled ears were chosen from six plants in a row at 4-m intervals. The sampled rows were chosen at $3-\mathrm{m}$ intervals but were at a slightly shorter interval near the donor boundary, as shown in Figure 4. Since an ear contained approximately 400 seeds and the average cross-pollination rate was calculated from six ears, the minimum detection level of the average cross-pollination rate of a row was $0.04 \%$, i.e., 1 part in 2400 .

\section{Characteristics of the model}

We conducted numerical experiments to predict pollen dispersal and cross-pollination by calculating the airflow and turbulence field using the A2Cflow model (Yamada Science and Art, Santa Fe, NM, USA) and then simulated pollen transport by A2Ct\&d (Yamada Science and Art). In this section, the model equations are described.

\section{Characteristics of model A2Cflow}

A2Cflow is a 3-D nonhydrostatic atmospheric model to predict atmospheric state. Flow dynamics are modeled by the Reynolds averaging equations and closed with twomoment turbulence closure equations to express turbulence around topography, buildings, or plant canopy. The predicted variables are three components of wind speed, air temperature, relative humidity, turbulent kinetic energy (TKE), turbulence length scale, and ground temperature. The prediction is made by numerically integrating momentum equations, a continuity equation, a thermodynamic equation, a radiative transfer equation, and a turbulence kinetic energy equation, using prescribed boundary conditions and initial conditions described later. The A2Cflow includes a canopy model module based on the work of Yamada (1982). For simplicity, a horizontally homogeneous canopy is assumed. The canopy model calculates drag force originating from the plant's leaves and stems to the momentum equations and a TKE equation.

The equations of mean motion are:

$$
\begin{aligned}
& \frac{\partial U}{\partial t}-f V=-\frac{1}{<p>} \frac{\partial p}{\partial x}+\frac{\partial}{\partial z}(-\overline{u w})-\eta C_{d} a(z) U|U| \\
& \frac{\partial V}{\partial t}+f U=-\frac{1}{<p>} \frac{\partial p}{\partial y}+\frac{\partial}{\partial z}(-\overline{v w})-\eta C_{d} a(z) V|V|
\end{aligned}
$$

where $U$ and $V$ are the components of the mean velocity of air in the $x$ and $y$ directions; $u, v$, and $w$ are velocity fluctuations in the $x, y$, and $z$ component; $f$ is the Coriolis parameter; $<p>$ is the horizontally averaged air density; $p$ is the pressure fluctuation; $\eta$ is a fraction of the area covered with plants, $0 \leq \eta \leq 1 ; C_{d}$ is a drag coefficient; and $a(z)$ is a plant's area density $\left(\frac{\mathrm{m}^{2}}{\mathrm{~m}^{3}}\right)$. The last term in equations (1) and (2) is the drag force from plants. Overbars indicate ensemble averages. A turbulence energy equation is given by

$$
\begin{array}{r}
\frac{\partial\left(\frac{q^{2}}{2}\right)}{\partial t}=\frac{\partial}{\partial t}\left[q l \tilde{S}_{q} \frac{\partial\left(\frac{q^{2}}{2}\right)}{\partial z}\right]-\overline{u w} \frac{\partial U}{\partial z}-\overline{v w} \frac{\partial V}{\partial z}+\beta \overline{w \theta_{v}} \\
-\frac{q^{3}}{B_{1} l}+\eta C_{d} a(z)\left[|U|^{3}+|V|^{3}\right]
\end{array}
$$

and a turbulence length scale, $l$, is obtained from

$$
\begin{aligned}
& \frac{\partial\left(q^{2} l\right)}{\partial t}=\frac{\partial}{\partial t}\left[q l \tilde{S}_{q} \frac{\partial\left(q^{2} l\right)}{\partial z}\right] \\
&-l E_{1}\left[-\overline{u w} \frac{\partial U}{\partial z}-\overline{v w} \frac{\partial V}{\partial z}+\beta g \overline{w \theta_{v}}\right]-\frac{q^{3}}{B_{1}}\left[1+E_{2}\left(\frac{l}{k z}\right)^{2}\right] \\
&+2 \eta C_{d} a(z) l\left[|U|^{3}+|V|^{3}\right]
\end{aligned}
$$

where $q^{2}=\overline{u^{2}}+\overline{v^{2}}+\overline{w^{2}}$ is twice the TKE, $\overline{w \theta_{w}}$ is a kinematic buoyancy flux, $E_{1}, E_{2}, \tilde{S}_{q}$ and $B_{1}=(1.8,1.33,0.2$, and 16.6) are empirical constants determined from laboratory experiments (Mellor and Yamada, 1982), $\beta$ is the coefficient of thermal expansion, $g$ is the gravitational acceleration, and $\theta_{v}$ is the fluctuation part of virtual potential temperature. The turbulent fluxes in equations (1)-(4) are obtained from simplified second-moment turbulence closure equations, $\left({ }^{-}(u w,)^{-} v w\right)=-l q S_{M}^{-}[\partial U / \partial z, \partial V / \partial z]$ and $\overline{w \theta}=-\alpha l q \tilde{S}_{M} \frac{\partial \theta}{\partial z}$, where $\tilde{S}_{M}$ and $\alpha$ are functions of the flux Richardson number, and $\alpha \equiv \frac{K_{H}}{K_{M}}$, where $K_{H}$ is an eddy diffusivity coefficient and $K_{M}$ is an eddy viscosity coefficient. 
The canopy model module also predicts heat exchange between plants and air originating from the radiative heating or cooling of plants. The net solar radiation $R_{N k}$ at a treetop is given by

$$
R_{N k}=\left(1-\alpha_{t}\right) S+R_{L h \downarrow}-R_{L h \uparrow}
$$

where $\alpha_{t}$ is the tree albedo, $S$ the direct solar radiation, $R_{L h \downarrow}$ is the incoming longwave radiation, and $R_{L h \uparrow}$ is the outgoing longwave radiation. $R_{L h \uparrow}$ is computed from

$$
R_{L h \uparrow}=\varepsilon_{t} \sigma T_{h}^{4}+\left(1-\varepsilon_{t}\right) R_{L h \downarrow}
$$

where $\varepsilon_{t}$ is the emissivity of trees, and $\sigma$ is the StefanBoltzmann constant. The net radiation in a canopy is assumed to be that given by Uchijima (1961),

$$
R_{N p}(z)=\eta R_{N k}\left[\exp \{-k L(z)\}-\eta\left(1-\frac{z}{h}\right) \exp \{-k L(0)\}\right]
$$

where $k$ denotes an extinction coefficient. The $L$ in the equation is a leaf area index

$$
L(z)=\int_{z}^{h}\left[a_{L}\left(z^{\prime}\right)+a_{s}\left(z^{\prime}\right)\right] \mathrm{dz}
$$

for which $a_{L}(z)$ is a leaf's surface area density $\left(\mathrm{m}^{2} / \mathrm{m}^{3}\right)$, $a_{S}(z)$ is a nonleaf surface's area density $\left(\mathrm{m}^{2} / \mathrm{m}^{3}\right)$, and $h$ is the height of a canopy. The second term in the parenthesis on the right-hand side of equation (7) is added to ensure that the net radiation vanishes at the ground when the surface is completely covered by plants $(\eta=1)$. The heatenergy balance equation within a canopy may be given as

$$
R_{N P}=H+L E
$$

where $H$ is the sensible heat flux and $L E$ is the latent heat flux. The internal heat energy equation is written as

$$
\frac{\partial \theta}{\partial t}=\frac{\partial}{\partial z}(-\overline{w \theta})+\frac{(1-\eta)}{\rho C_{p}} \frac{\partial R_{N}}{\partial z}+\frac{\eta}{\rho C_{p}}\left(1+\frac{1}{B}\right)^{-1} \frac{\partial R_{N P}}{\partial z}
$$

where the Bowen ratio $(=H / L E)$ in a canopy is assumed to be constant with height. The last term on the right-hand side of equation (10) is obtained first by differentiating (7) with respect to $z$ and then substituting $L(z)$ from (8). A conservation equation for the mixing ratio of water vapor is given by

$$
\frac{\partial Q_{v}}{\partial t}=\frac{\partial}{\partial z}\left(-\overline{w q_{v}}\right)
$$

where $q_{v}$ and $Q_{v}$ are the mean and fluctuation of the water vapor mixing ratio, respectively. The source of water vapor due to evapotranspiration of plants is ignored in the present study.
Surface boundary conditions for equations (1), (2), (3), (4), (10), and (11) are constructed from the empirical formulas by Dyer and Hicks (1970) for the nondimensional wind and temperature profiles (details are found in the Appendix in Yamada (1981)). The soil temperature is obtained by solving a heat conduction equation with heat energy balance (radiative, sensible, and latent heat fluxes) at the soil surface; the soil temperature is obtained at a depth of $50 \mathrm{~cm}\left(25^{\circ} \mathrm{C}\right.$ in this study). Boundary conditions along the upper computational boundary for $U$ and $V$ are defined by geostrophic wind, and turbulence is assumed to vanish.

Cloud microphysical parameters such as clouds or rainwater amounts are not calculated in this experiment. The nonhydrostatic option is based on the work of Hirt and Cook (1972). Details of the governing equations can also be found in Yamada and Bunker (1988) as HOTMAC.

\section{Characteristics of model A2CtEd}

A2Ct\&d is a random particle statistical diffusion model. It predicts the turbulent diffusion transport of tracer particles using the airflow field predicted by A2Cflow through a Lagrangian form.

In this study, we ignored pollen deposition on the plants and pollen resuspension for simplicity. We mainly focused on the cross-pollination rate calculated from the ratio of donor and recipient pollen concentrations, which would not be changed by deposition and resuspension rates on the plants, as was shown in Dupont et al. (2006) and supported by Klein et al. (2003), who argued that excluding pollen deposition on plants yielded better prediction of cross-pollination.

Locations of particles are computed from

$$
x_{i}(t+\Delta t)=x_{i}(t)+U_{p i} \Delta t
$$

where

$$
U_{p i}=U_{i}+u_{i}
$$

$$
\begin{gathered}
u_{i}(t+\Delta t)=a u_{i}(t)+b \sigma_{u_{i}} \zeta+\delta_{i z}(1-a) t_{L x_{i}} \frac{\partial}{\left(\partial x_{i}\right)\left(\sigma_{u_{i}}^{2}\right)} \\
a=\exp \left(-\frac{\Delta t}{t_{L x_{i}}}\right) \\
b=\left(1-a^{2}\right)^{\frac{1}{2}} .
\end{gathered}
$$

In the above expressions, $U_{p i}$ is the particle velocity in the $x_{i}$ direction, $\zeta$ is a random number from a Gaussian distribution with zero mean and unit variance, $t_{L x_{i}}$ is the Lagrangian integral time for the velocity $u_{i}, \sigma_{u_{i}}$ is the standard deviation of velocity fluctuation $u_{i}$, and $\delta_{i z}$ is 
the Dirac delta. The mean velocity, $U_{i}$, and the standard deviation of wind, $\sigma_{u_{i}}$, are obtained from the A2Cflow model. The last term in equation (14) is introduced in order to correct for the accumulation of particles into lower turbulent energy regions. The Lagrangian time scales, $t_{L z}=20 \mathrm{~s}$ and $t_{L x}=t_{L y}=10^{4} \mathrm{~s}$, were used in this study.

Theoretically, to determine particle concentration at a given time and location by counting the number of particles, an infinite number of particles should be calculated to describe a smooth distribution. To produce a smooth distribution by calculating a finite number of particles, a "kernel" density estimator is used (Yamada and Bunker, 1988) where each particle represents a center of the puff, expressing a Gaussian distribution of concentration, for which variances are determined by the time integration of the velocity variances encountered over the history of the puff. The concentration level at a given time and location is determined as the sum of the concentrations each puff contributes.

Concentration $\chi$ at $(X, Y, Z)$ is estimated by using the following expression:

$$
\begin{aligned}
& \chi(X, Y, Z)=\frac{Q \Delta t}{(2 \pi)^{\frac{s}{2}}} \sum_{K=1}^{N} \frac{1}{\sigma_{x k} \sigma_{y k} \sigma_{z k}} \\
& \times \exp \left(-\frac{1}{2} \frac{\left(x_{k}-X\right)^{2}}{\sigma_{x k}^{2}}\right) \exp \left(-\frac{1}{2} \frac{\left(y_{k}-Y\right)^{2}}{\sigma_{y k}^{2}}\right) \\
& \times\left\{\exp \left(-\frac{1}{2} \frac{\left(z_{k}-Z\right)^{2}}{\sigma_{z k}^{2}}\right) \exp \left(-\frac{1}{2} \frac{\left(z_{k}+Z-2 z_{g}\right)^{2}}{\sigma_{z k}^{2}}\right)\right\}
\end{aligned}
$$

where $\left(x_{k}, y_{k}, z_{k}\right)$ is the location of the $k$ th particle; $\sigma_{x k}$, $\sigma_{y k}, \sigma_{z k}$ are standard deviations of a Gaussian distribution; and $z_{g}$ is the ground elevation. The variances are approximated; for example, $\sigma_{y}$ is approximated as

$$
\begin{aligned}
& \sigma_{y}=\sigma_{v} t \text { for } t \leq 2 t_{L y} \\
& \sigma_{y}^{2}=2 t_{L y} \sigma_{v}^{2} t \text { for } t>2 t_{L y} .
\end{aligned}
$$

More details have been described in Yamada and Bunker (1988) as RAPTAD.

\section{Model configuration}

Figure 12 introduces the numerical prediction process used in this study. First, the simulated model domain of A2Cflow was configured as in the broken-dot line in Figure 1. Each domain (A, B, and C) was calculated separately to predict the wind field and pollen transport as precisely as possible given the limited computer resources. Topography was neglected, and an average altitude of $1300 \mathrm{~m}$ was applied for simplicity. The numerical model domain comprises a $75 \times 40 \times 51$ grid in an $x-y-z$ coordinate system with a horizontal grid interval of $1.5 \mathrm{~m}$.
The vertical grid interval was $0.3 \mathrm{~m}$ below $8 \mathrm{~m}$ and gradually increased up to $23 \mathrm{~m}$ at the upper boundary of $300 \mathrm{~m}$. The vertical grid interval of $0.1 \mathrm{H}$ and the horizontal grid interval of $0.5 \mathrm{H}$ were sufficient for calculating the effects of windbreaks where $H$ was the height of the windbreak (Wang and Takle, 1995). Therefore, those grid intervals were adequate for the 6-m-tall windbreak net. For the maize and sorghum canopies, whose heights were less than $2 \mathrm{~m}$, the criteria were not strictly satisfied but were near to the optimum criteria.

The effects of the maize and sorghum canopies were numerically expressed by the canopy model; canopy heights are indicated in Table 1. The leaf surface area density $a_{L}(z)$ of the maize and the surrounding sorghum, $a_{L}(z)$, used in equations (1)-(4) and (8), was assumed to have a maximum of $1.0 \mathrm{~m}^{-1}$ at $0.9 h$ ( $h$ is the height of a canopy, as in equation (8)) and linearly decreased to $0 \mathrm{~m}^{-1}$ at $h$ and $0.1 h$. The leaf surface area density of the sorghum planted between the donor and recipient plots in domain $\mathrm{C}$ was defined in the same way, but its maximum was $5.0 \mathrm{~m}^{-1}$ because the sorghum plot was grown as a dense plot. The nonleaf surface area density, $a_{s}(z)$, was defined uniformly as $0.05 \mathrm{~m}^{-1}$. $C_{d}$ of maize and sorghum was set at 0.2 . The canopy model was also used to express the drag force of the windbreak net. For this purpose, $C_{d}$ and $a_{L}(z)+a_{s}(z)$ were set uniformly at all heights to 1.02 and $10.0 \mathrm{~m}^{-1}$, respectively, which were obtained from wind tunnel measurements (Ushiyama et al., 2009). In the case of the 2-mm-mesh net in the additional numerical experiment, $C_{d}$ and $a_{L}(z)+a_{s}(z)$ were set uniformly to 0.57 and $1.0 \mathrm{~m}^{-1}$, respectively (Ushiyama et al., 2009).

Time integrations were conducted from 6:00 to 18:00 local time (LT) each day from Aug. 15 to 31, 2007. The 3-hourly horizontal wind speed $(u, v)$, temperature $(T)$, and relative humidity $(R H)$ from the GPV data produced by the Japan Meteorological Agency were assimilated into the model variables. The GPV had a horizontal resolution of 0.125 degrees in the east-west direction and 0.1 degrees in the north-south direction (approximately $10 \mathrm{~km}$ ), with vertical grid points at the surface, pressure level of $1000 \mathrm{hPa}, 975 \mathrm{hPa}, 950 \mathrm{hPa}, 925 \mathrm{hPa}, 900 \mathrm{hPa}$, $850 \mathrm{hPa}, 800 \mathrm{hPa}$, and so on. Thus, the data gave a fairly good reproduction of realistic meteorological fields at a cloud scale. The GPV was interpolated on the A2Cflow model grids and nudged into all the grid points of the model instead of forcing them as boundary conditions. The nudging factor was $10^{-4}$ at the model's upper boundary and decreased linearly to zero at the ground because the evolution of turbulence in the model was more important near the ground than forcing a match to the GPV data. Cloud amount data in the GPV were also used to modify radiative forcing. The average hourly horizontal wind speed observed by two AWSs in each domain was also assimilated into the model to adjust the wind as 


\section{Flow chart of the prediction of maize pollen dispersal and cross-pollination distribution}

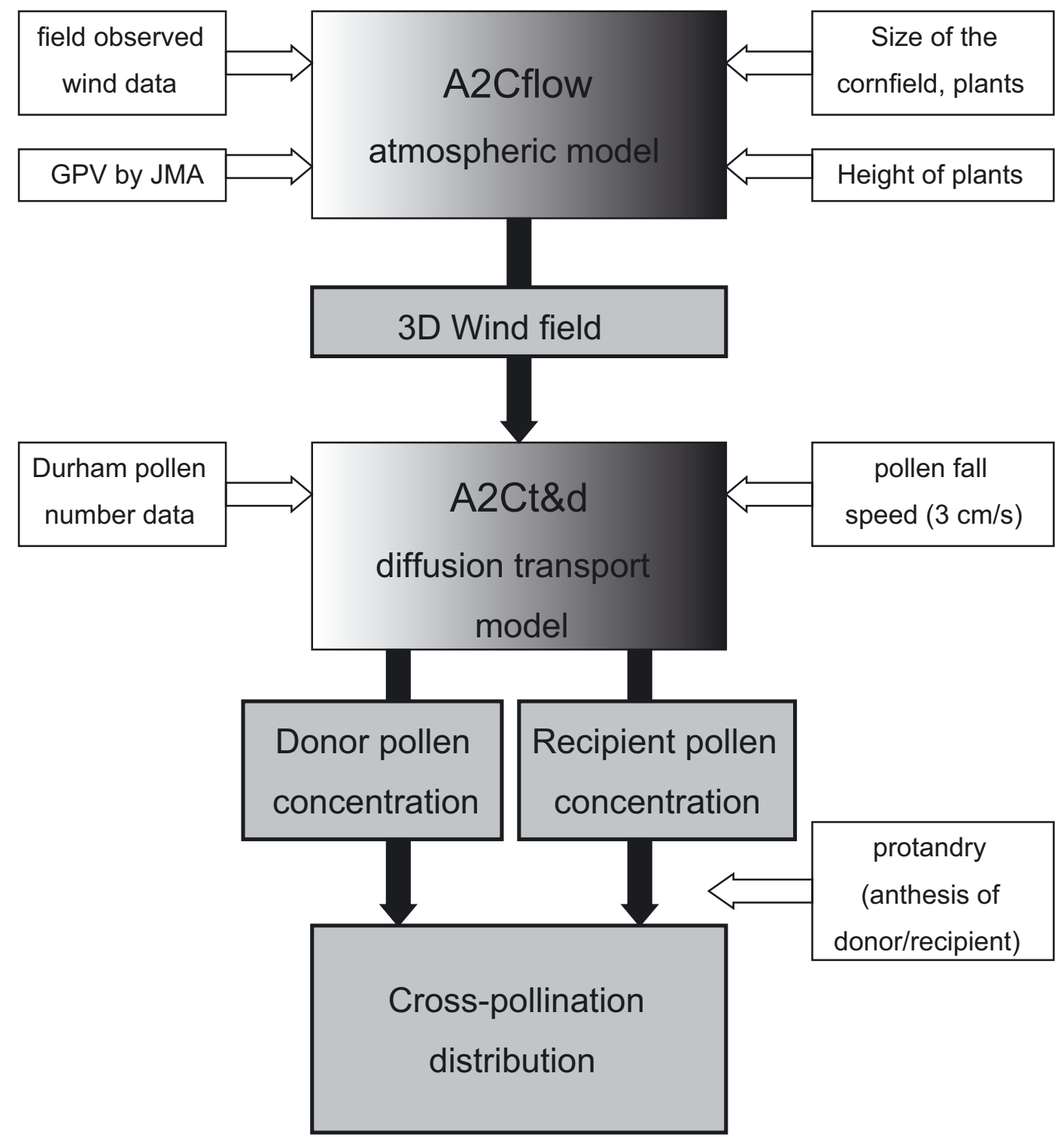

Figure 12. Flow chart of the numerical predictions.

observed with a nudging factor of $10^{-4}$ at the height of $2.5 \mathrm{~m}$. Time intervals of integrations were automatically determined in the model and were $0.1-0.5 \mathrm{~s}$.

For the calculation by A2Ct\&d, data of A2Cflow at 30-min intervals were used. The pollen emission rates were required in the pollen dispersion calculation. We used the pollen accumulation data (Fig. 2) to deter- mine daily emitted pollen numbers. To determine the diurnal variation, we invoked the time derivative of the air temperature. The pollen emission rate of maize increases rapidly after sunrise, and it decreases gradually after late morning (Jarosz et al., 2005; Kawashima et al., 2004, 2007). As shown in Kawashima et al. (2004), the pollen emission rate from maize tassels is associated with 
the temporal variation in the air temperature. In this study, the pollen emission rate was determined by the time derivative of the air temperature at a grid point near the tassels. The total pollen number emitted in 1 day was later adjusted using the observed number for the respective day and domain, as shown in Figure 2. In a single continuous calculation from 6:00 LT to 18:00 LT, approximately 5000 kernels were emitted from both the donor and recipient plots. The locations of the kernel emissions were given by a random function in each plant area (Fig. 1), and the heights of emission were assumed at the top of the canopy summarized in Table 1.

The A2Ct\&d algorithm also requires information about the deposition speed of maize pollen. Aylor (2002) estimated the settling speed of maize pollen to be $0.32 \mathrm{~m} . \mathrm{s}^{-1}$ when pollen is fresh and wet, and $0.21 \mathrm{~m} . \mathrm{s}^{-1}$ when it is dry and dehydrated. In this study, the dispersal distance was not far (50 m at most); therefore, the pollen was assumed to be fresh. We used a settling speed of $0.30 \mathrm{~m} \cdot \mathrm{s}^{-1}$. Maize pollen maintains its viability for at least a few hours (Loos et al., 2003). Therefore, we assumed that all the pollen grains were viable in our calculation.

After the calculation of the pollen dispersal, the crosspollination rate $(C)$ within the recipient area was calculated from the donor $\left(N_{D}\right)$ and recipient pollen concentrations $\left(N_{R}\right)$ at the height of the ears $(0.75 \mathrm{~m})$ as

$$
C=\frac{\sum\left(N_{D_{i}} N_{R_{i-2}}\right)}{\sum N_{R_{i-2}} \sum\left(N_{D_{i}}+N_{R_{i}}\right)}
$$

where $i$ indicates each day. In the calculation, a weighting average with respect to the amount of recipient pollen was applied to take into account the effect of anthesis. Generally, maize is a protandrous species, i.e., pollen shedding begins several days before silking. The protandry score varies from 0 to 5 days depending on the combination of drought stress and plant density (Tab. 2 in Angevin et al., 2008). Because of the low plant density $\left(53200 \mathrm{ha}^{-1}\right)$ and moderate drought stress of this experiment, the protandry score was 2 days. In the numerator and denominator of equation (1), the weighting average coefficient, $N_{R}$, has the subscript $i-2$, which means the weighting average was applied with respect to the pollen number of the recipient 2 days prior to the calculated day as defined by the protandry score.

\section{ACKNOWLEDGEMENTS}

The authors thank Dr. Tetsuji Yamada for his valuable advice and suggestions throughout the analysis. We also thank the technical officials and staff at the Tsumagoi Station of the National Center for Seeds and Seedlings for their efforts in maintaining the cornfield and obtaining the pollen dispersal and cross-pollination rate data. The GFD Dennou Club Library and Grid Analysis and Display System (GrADS) were used to draw the figures. The GPV analysis data were produced by the Japan Meteorological Agency and reformatted into NetCDF by the Research Institute for Sustainable Humanosphere, Kyoto University.

Received August 15, 2009; accepted June 20, 2010.

\section{REFERENCES}

Angevin F, Klein EK, Choimet C, Gauffreteau A, Lavigne C, Messean A, Meynard JM (2008) Modeling impacts of cropping systems and climate on maize cross-pollination in agricultural landscapes: The MAPD model. Europ. J. Agronomy 28: $471-484$

Aylor DE (2002) Settling speed of corn (Zea mays) pollen. J. Aerosol Sci. 33: 1601-1607

Aylor DE, Schultes NP, Shields EJ (2003) An aerobiological framework for assessing cross-pollination in maize. Agric. For. Meteorol. 119: 111-129

Aylor D, Boehm MT, Shields EJ (2006) Quantifying areal concentration of maize pollen in the atmospheric surface layer using remote-piloted airplanes and Lagrangian stochastic modeling. J. Appl. Meteor. 45: 1003-1015

Dupont S, Brunet Y, Jarosz N (2006) Eulerian modeling of pollen dispersal over heterogeneous vegetation canopies. Agric. For. Meteorol. 141: 82-104

Dyer AJ, Hicks BB (1970) Flux-gradient relationship in the constant flux layer. Quart. J. Roy. Meteor. Soc. 96: 715-512

Helbig N, Vogel B, Vogel H, Fiedler F (2004) Numerical modeling of pollen dispersion on the regional scale. Aerobiologia 3: 3-19

Hirt CW, Cook JL (1972) Calculating three-dimensional flows around structures and over rough terrain. J. Comput. Physics 10: 324

Jarosz N, Loubet B, Huber L (2004) Modelling airborne concentration and deposition rate of maize pollen. Atmos. Environ. 38: 5555-5566

Jarosz N, Loubet B, Durand B, Foueillassar X, Huber L (2005) Variations in maize pollen emission and deposition in relation to microclimate. Environ. Sci. Technol. 29: 43774384

Jia S, Wang F, Sui L, Yuan Q, Liu W, Liao Y, Li S, Jin W, Peng H (2007) Transgene flow to hybrid rice and its malesterile lines. Transgenic Res. 16: 491-501

Kawashima S, Fujita T, Matsuo K, Shibaike H (2004) Development of an automatic corn pollen monitor. Jpn. J. Palynol. 50: 5-14

Kawashima S, Matsuo K, Shibaike H, Takahashi Y, Inoue S, Yonemura S, Du M (2007) Effects of biological and meteorological conditions on inter-annual variation in hybrid percentage of maize. Jpn. J. Palynol. 53: 9-17 


\section{T. Ushiyama et al.}

Klein EK, Lavigne C, Foueillassar X, Gouyon P, Laredo C (2003) Corn pollen dispersal: quasi-mechanistic models and field experiments. Ecol. Monogr. 73: 131-150

Kuparinen A (2006) Mechanistic models for wind dispersal. Trends Plant Sci. 11: 296-301

Kuparinen A, Markkanen T, Riikonen H, Vesala T (2007) Modelling air-mediated dispersal of spores, pollen, and seeds in forested areas. Ecol. Model. 208: 177-188

Loos C, Seppelt R, Meier-Bethke S, Schiemann J, Richter O (2003) Spatially explicit modeling of transgenic maize pollen dispersal and cross-pollination. J. Theor. Biol. 225: 241-255

Mellor GI, Yamad T (1982) Development of a turbulence closure model for geophysical fluid problems. Rev. Geophys. Space Phys. 20: 851-875

Messean A, Angevin F, Gomez-Barbero M, Menrad K, Rodriguez-Cerezo E (2006) New case studies on the coexistence of GM and non-GM crops in European agriculture, Technical Report EUR No: 22102 EN

Nathan R, Schurr FM, Spiegel O, Steinitz O, Trakhtenbrot A, Tsoar A (2008) Mechanisms of long-distance seed dispersal. Trends. Ecol. Evol. 23: 638-647

Okubo A, Levin SA (1989) A theoretical framework for data analysis of wind dispersal of seeds and pollen. Ecology 70: 329-338

Pasken R, Pietrowicz JA (2005) Using dispersion and mesoscale meteorological models to forecast pollen concentrations. Atmos. Environ. 39: 7689-7701

Rognli O, Nilsson N-O, Nurminiemi M (2000) Effects of distance and pollen competition on gene flow in the windpollinated grass Festuca pratensis Huds. Heredity 85: 550560

Schueler S, Schlünzen KH (2006) Modeling of oak pollen dispersal on the landscape level with a mesoscale atmospheric model. Environ. Model Assess. 11: 179-194

Stauffer DR, Seaman NL (1990) Use of four-dimensional data assimilation in a limited-area mesoscale model. Part I: Experiments with synoptic-scale data. Mon. Wea. Rev. 118: $1250-1277$
Uchijima Z (1961) On characteristics of heat balance of water layer under paddy plant cover. Bull. Nat. Inst. Agric. Sci. A: 243-263

Ushiyama T, Inoue S, Shibaike H (2009) Measurements of wind suppression effects of windbreak net using a wind tunnel for the purpose of applying numerical simulations. J. Agric. Meteorol. 65: 273-281

Wang H, Takle ES (1995) A numerical simulation of boundary-layer flows near shelterbelts. Boundary-Layer Meteorol. 75: 141-173

Wang TY, Chen HB, Reboud X, Darmency H (1997) Pollenmediated gene flow in an autogamous crop: Foxtail millet (Setaria italica). Plant Breed. 116: 579-583

Wilson JD, Sawford BL (1996) Review of Lagrangian stochastic models for trajectories in the turbulent atmosphere. Boundary-Layer Meteorol. 78: 191-210

Yamada T (1981) A numerical simulation of nocturnal drainage flow. J. Meteor. Soc. Jpn. 59: 108-122

Yamada T (1982) A numerical model study of turbulent airflow in and above a forest canopy. J. Meteor. Soc. Jpn. 60: 439-454

Yamada T (2000) Numerical simulations of airflow and tracer transport in the southwestern United States. J. Appl. Metor. 39: 399-411

Yamada T, Bunker S (1988) Development of a nested grid, second moment turbulence closure model and application to the 1982 ASCOT Brush Creek data simulation. J. Appl. Meteor. 27: 562-578

Yamada T, Bunker S, Moss M (1992) Numerical simulations of atmospheric transport and diffusion over coastal complex terrain. J. Appl. Meteor. 31: 565-578

Yao K, Hu N, Chen W, Li R, Yuan Q, Wang F, Qian Q, Jia S (2008) Establishment of a rice transgene flow model for predicting maximum distances of gene flow in southern China. New Phytol. 180: 217-228

Zar JH (1984) Biostatistical analysis, second edition, Prentice Hall, London, pp 493 